\title{
Evolutionary Conception in Snow Cover Science
}

\author{
E.G. Kolomyts \\ Institute of Ecology of the Volga River Basin, Russian Academy of Sciences, 10, Komzina str., Togliatti, 445003, Russia \\ *Corresponding Author: egk2000@mail.ru
}

Copyright $@ 2014$ Horizon Research Publishing All rights reserved.

\begin{abstract}
The report presents novel approach to the study of development of snowpack structure based on its crystal morphology and the fundamental laws of natural symmetry. It is worked out the empirical deterministic models describing the sublimation-metamorphic cycle of seasonal snow cover and the polymorphic variants of this cycle. Stadiality of the forms of crystal growth and self-development snow layers are revealed. They are a result of successive process of superposition of ice crystal-chemical symmetry and dissymmetry of the whole system soil-snow-atmosphere, according to the known P. Curie's principle. Crystal-morphological classification for season snow is worked out by author on the base of the given an account evolutionary model. Evolution of snowpack structure is conditioned to a marked degree by probabilistic conformity to natural laws, manifesting themselves in the processes of auto-regulation of metamorphism. These processes include the two types of regulation: the self-regulation of snow layers, on the one hand, and their regulation from outside - under the influence of atmospheric perturbations, on the other hand. An analysis of the processes of auto-regulation of metamorphism is capable of rendering a substantial service in the development of methods of shortand long-term forecasting of the avalanches.
\end{abstract}

Keywords Snow Cover, Structure, Crystal-Morphology, Symmetry Theory, Evolution, Auto-Regulation, Classification For Snow

\section{Introduction}

Up to the present, the study of processes within snow packs has been based on consideration of snow cover mainly as a continual matter-energy system. It has already become a tradition to phenomenological approach (according to classic thermodynamic) - to interpret snow on the ground as a three-component porous material capable of irreversible viscous deformations [Watanabe, 1980; Edens and Brown, 1991, Golubev, Frolov, 1998; et al.] and reflecting the winter hydro-meteorological conditions [Colbeck, 1982; Durand et al., 1993; Bartelt et al., 2002; Lhening et al., 2002; etc.]. In this respect, the most important physical-mechanical properties of snow have been studied completely enough [Gray, Mail, 1981; Pielmeier and Scheebeli, 2003]. Their mathematical models have been developed, which are of great theoretical and applied significance, first of all, for prediction of snow avalanches [Brun et al., 1992; Bartelt , Lehning, 2002; Bozhinsky et al., 2002; Lehning et al., 2002]. The systems of classification by the character and degree of avalanche hazard have been developed for the mountain territories of the former USSR on the basis of coupled analysis of geophysical processes and geographical avalanche-formation factors [Bozhinsky, Losev, 1978].

At the same time, in most of the snow studies (both in Russia and abroad) the methods of contemporary crystal-morphology are still insufficiently employed in structural snow science and avalanche science, thus to some extent retarding further development of these branches of glaciology. It is true that the modern studies into the structure of snow cover consider it as a heterogeneous environment varying in time in its structural and stratigraphic characteristics [Sokratov, 2001; Bartelt, Lehning, 2002; Chernov, 2003; Kaempfel et al., 2004; Schneebeli, Sokratov, 2004; etc.]. However, here it is necessary to search for regularities of these variations and to establish the mechanisms controlling evolutionary transformations of snow structures. The question of the extent to which the sublimation metamorphism of deposited snow is a process of its self-development, i.e., a really evolutionary process in the strict scientific sense of this term [Shmalgauzen, 1969; Timofeev-Resovsky et al., 1977; Odum, 1983], is still open. What is meant here is directional structural changes in a given natural system, first, from simple to complex and, second, on the basis of primarily internal interactions in the system, against the background of its adaptation to variable environment.

In the very beginning of snow cover studies in the regions of Siberia and Far East the author [Kolomyts, 1976] encountered the poor information that could be obtained using already established methods of field structure analysis of seasonal snow. Snow cover is first of all a crystalline body, and hence the processes of its metamorphism obey the basic laws of crystallography [Vernadsky, 1965; Shafranovsky, 1968] which unfortunately are little employed by glaciologists in analyzing snow structure in the field and in interpreting the data obtained. At the same time one can 
derive only very limited data on the processes occurring in the snowpack using generally accepted methods of structural analysis of snow sections in nature. That is way common snow structure analysis are usually based on the methods of granulometry [Seligman, 1936; Golubev, Frolov, 1998; Brown and Edens, 1991; Brun et al., 1992; etc.], while the forms of crystals are considered as the essential but not main structural-stratigraphic property of snow cover [Schaefer et al., 1954; Gray, Mail, 1981; Colbeck et al., 1990; Bartelt, Lehning, 2002]. Last "International Classification for Seasonal Snow on the Ground" [Fierz et al., 2009] has made essentially step forwards as compared with the previous classifications [Schaefer et al., 1954; Sommerfeld and LaChapelle, 1970; Colbeck et al., 1990] in respect of further equipment of methodical arsenal for the field structure research of snow cover.

The term "evolution of snowpack" is used pretty often [Colbeck, 1982; Lehning et al., 2002; Pielmeier and Schneebeli, 2003; Kaempfer et al., 2004; Sokratov, Troshkina, 2009; etc.], however it has no particular meaning so far. It is still unclear to what extent the metamorphism of packed snow is a process of its self-development, i.e., a really evolutionary process, in the strict scientific meaning of this term. In the meantime, the whole history of West-European and Russian snow studies [Seligman, 1936; Paulke, 1938; Gray, Mail, 1981; Tushinsky et al., 1953; Kolomyts, 1977, 1984; etc.] shows that under some or other winter conditions snow cover is transformed from disordered pile of snow crystals deposited from the atmosphere into something integral, ordered, organized, i.e., creates itself as a certain glaciosystem. It may be a priori affirmed that the theory of evolution is fully applicable to metamorphic transformations of seasonal snow cover.

The morphology of snow crystals is studied mainly by means of laboratory experiments and observations of their individual free growth and morphogenesis [Brown and Edens, 1991; Tushinsky et al., 1953; Nakaya, 1954; Kobayashy, 1957; Yosida, 1966; etc.], which substantially helps interpret the results of on-site observations. However, it is very difficult to reproduce in laboratory conditions the full nature diversity of evolutionary metamorphism processes in snow cover.

The centuries-old experience of establishment of the evolution theory in biology has shown that only discrete objects (individuals, species) and their system-forming combinations (populations, communities) as discrete qualitatively definite formations can evolve, but not some continual characteristics of individuals, populations and communities. In snow cover, such qualitative definiteness is typical only of crystal forms and a kind of their communities but in no way of grain sizes, parameters of grain contacts, surface energy, etc. (i.e., not granulometric characteristics). Grains cannot evolve (in the strict sense of this term) by definition. We believe that if it is excluded from consideration such a most important structural characteristic as the form of growing or evaporating crystals embodying the most structural quality of snow, then it makes no sense to speak about snow cover evolution as a directional process of its metamorphism.

Thus, glaciological engineering so far has not a sufficiently elaborated scientific and methodical basis for predicting the whole class of avalanches of according to terminology [Tushinsky et al., 1953], or, avalanches of prolonged development, by definition [Bozhinsky, Losev, 1978], associated mainly with the evolutionary processes of metamorphism in snow cover.

It is necessary to elaborate an evolutionary basis of the theory of sublimation metamorphism of seasonal snow cover for improvement of the scientific and methodical basis of snow avalanche prediction and development of the methods of indication of the winter regime of landscapes by snow structure. Solution of this problem is envisaged by way of constructing discrete models of system organization and development of snow cover based on its crystal morphology and fundamental laws of natural symmetry, as well as developing on the basis of these models the methods for investigation of the processes of sublimation snow metamorphism from positions of the theory of evolution. This theory, formed within biological sciences [Timofeev-Resovsky et al., 1977], has been effectively applied to crystallography and genetic mineralogy [Shafranovsky, 1968; Shubnikov, 1975; Yushkin, 1977], as snow cover is a monomineral rock [Rikhter, 1955]. It should be proved that the transformation of evolutionary units of snow cover (shapes of crystals and their communities) is based on their self-development (self-organization and autoregulation), which has both invariant and stochastic regularities, as well as the properties of adaptation to the varying winter meteorological regime. All these characteristics of snow cover sublimation metamorphism correspond to the propositions of the general theory of evolution of natural systems.

\section{Starting Position for the Evolutionary Snow Studies}

By the author's earnest conviction, evolutionary snow studies must be based on the probabilistic-statistical approach considering snow cover as a hierarchically organized system of discrete crystal spaces as interpreted by V.I. Vernadsky (1965). This system is based on the key structural property of snow: the shapes of growing crystals that reflect the whole complex of internal transformations of deposited snow. We postulate that snow cover is the system of natural assemblages of crystal forms, what in sometimes sense are analogous to the communities of individuals with different quality in living nature, according to definition [Shubmikov, Koptsik, 1972; Yushkin, 1977]. New snow crystals grow in a close mutual interaction and simultaneously experience the outside regulatory control, first of all from the atmosphere. With such approach, the analysis of snow structure and morphology must be based on the methods of crystal-morphology and fundamental laws of 
natural symmetry.

The transformation of sedimentary rock into metamorphic is bound up with its secondary changes (metagenesis) that occur with sudden changes in the external medium. Metamorphic changes lead to partial or complete re-crystallization of the rock with formation of new structures [Paffengolts, 1973]. If in this process there is only decomposition of the initial crystals and their aggregates, the process is referred to as destructive metamorphism; if there is new crystal growth it is constructive metamorphism [Kuznetsov, 1953; Quervain de, 1958]. When one talks about the re-crystallization of snow as one of the basic processes of its metamorphism, according to P. A. Shumskiy (1955), one should bear in mind that sublimation re-crystallization, that is the growth of new crystals through the vaporous phase, is major matter-energy process, creating the most natural diversity of snow cover structures. The sublimation re-crystallization of snow relates to a type of hydrothermal metamorphism of rocks that is caused by the ascending motions of gaseous flows (water vapors) from the warmer to the colder layers (as a rule upwards).

The basic law of crystallography states that crystal forms represent the processes of heat and moisture transfer in the medium surrounding them, and all conformity to natural laws of the growth of real crystals in essence characterize their divergence from the equilibrium state conforming to the internal (crystal-chemical) structure of the given material. This concerns the staged adaptation of a growing symmetrical single crystal to an dissymmetric medium according to the well-known principle of the superposition of symmetries of Pierre Curie (1908), which is regarded as symmetric interpretation of the second law of thermodynamics [Shubnikov, Koptsik, 1972]. The principle of superposition of two symmetries, that is leave traces on each other, in the most general form appears as:

\section{phenomenon $($ effect $)=$ characteristic $\times$ action .}

In accordance with Curie's principle, snow structure development is a process irreversible in time, which consists of successive stages of superposition (overlap) of hexagonal and trigonal symmetry of ice as a mineral (genotype) and dissymmetry of the vector hydrothermal field of snowpack, as well as the field of relaxation of mechanical stress in the latter. It results in formation of a genetically integral chain of real ("forced", false) crystal forms (phenotypes) as a way of adaptation of growing crystals to environment [Sheftal, Kolomyts, 1973; Shafranovsky, Plotnikov, 1975].

By the author's earnest conviction, evolutionary snow studies must be based on the probabilistic-statistical approach considering snow cover as a hierarchically organized system of discrete crystal spaces [Kolomyts, 1976, 1984]. This system is based on the key structural property of snow: the shapes of growing crystals that reflect the whole complex of internal transformations of deposited snow. We postulate that snow cover is the system of natural assemblages of crystal shapes, what in sometimes sense are analogous to the communities of individuals with different quality in living nature, according to definition [Shubnikov, Koptsik, 1972; Yushkin, 1977].

\section{Objects and Methods}

The author put forward a concept of the integral unclosed sublimation-metamorphic cycle of dry snow and its polymorphic (regional) versions. This concept was first substantiated by the data from the author's independent stationary studies on experimental plots in winters 1966/1967-1969/1970 in West Siberian middle taiga and in the low-mountain taiga of the Lower By-Amur in winter 1976/1977. Then it was confirmed during his route snow-measuring works in other plain and mountain regions of Russia: in the Center and North-West of the Russian Plain (Moscow and Ladoga regions), in the Khibini Mountains, in the Middle Priamurye and Primorye, in the Polar Urals, on the South Sakhalin and Kamchatka, in the mountain regions of the West Sayan and Verkhoyanye (the upper Kolyma river basin) and, finally, in the highlands of the Central Caucasus (Prielbrusye - By-Elbrus). It has been convincingly shown that the sublimation-metamorphic transformations of each snow pack layer in time are observed in nature as a spontaneous, genetically uniform chain of transformations of some crystal shapes into other, with the respective change of the structure of crystal communities in each layer. Such a chain of invariant metamorphic events includes certain steps (the periods of metamorphism), which in turn are divided into crystal growth stages and age-related phases of snow layer metamorphism. It is in agreement with the thesis known from mineralogical crystallography: any crystal individual, before it becomes what it is, passes through certain growth stages.

In the time of stationary observations of snow structure and hydrothermal regime the information was registered during winter in on and the same snow sections with an interval of about one month. At the end of winter seasons of the same years, the route studies of snow structure were performed in different plain and mountain regions of Russia: from extracontinental (Kolyma Region) to suboceanic (West Greater Caucasus Region).

Based on the known concepts of the laws of real crystallization [Honigman, 1958; Shafranovsky, 1968; Sheftal, 1973; Yushkin, 1977; et al.], the forms of crystal growth and destruction were determined for each genetic layer of snow pack. Multiple determinations of crystal shapes and particle measurements were performed at a 5-10-fold optical magnification within the accuracy of 0.1 $\mathrm{mm}$. The statistical analysis of empirical material has shown that the probability of the final result varies within $70-75 \%$ per each snow sample for 200-300 measurements, i.e., quite permissible accuracy for field studies.

The following numerical parameters of snow structure were obtained: the average radius and volume of crystals, the percentage of shapes in each layer, the number of crystals per volume unit, the effective surface and area of cohesion of 
crystals, the layer-by-layer snow density, and the average weight of a single crystal. The crystal-morphological method yielded mass quantitative data on the processes and results of snow metamorphism under field conditions, without using complex facilities.

Formalized description of snow cover as an integral community of affine individuals (crystal shapes) was prepared using the variation and dispersion analyses, the elements of the descriptive fuzzy set theory and the information theory. The new crystals growing in snow pack acquire certain interrelationships: genetic, spatial, regular, etc., i.e., the snow cover during metamorphism acquires certain organization (situational orderliness), which we consider as a synonym of the concept of "the degree of sublimation metamorphism of snow". This degree was estimated by the value of proposed snow cover re-crystallization coefficient [Kolomyts, 1976, 1977].

"Crystal growth in nature is not altogether unvaried growth" [Grigoryev, 1965, p. 70]. The primary forms of secondarily idiomorphic crystals examined above are the result of varied processes of constructive metamorphism in the snow cover. Every snow layer exposed to such transformation acquires some set of forms of secondary crystals with corresponding dimensions, that is, it is characterized by a particular crystal-morphological complexity. This set of varied forms, according to W. R. Eshby's concept (1956), may be represented as a set of their probabilities (chances of being encountered) adding up to unity. Probability serves as the structural characteristic of the system because a change in probability leads to a change in the linkages between elements. The variety of crystal forms represented in values of probability constitutes the linked (structural) information contained in each snow layer.

From a formal standpoint structural information is analogous to the negative entropy of physical systems (Boltzmann's entropy as a measure of homogeneity, chaos), and hence it is referred to as negentropy [Brillouin, 1956]. It may be looked upon as a function of the state of cybernetic systems and also as a parameter of their complexity.

If a given volume of snow cover contains an $n$-th quantity of classes of crystal forms $\left(\mathrm{F}_{1}, \mathrm{~F}_{2}, \ldots, \mathrm{F}_{n}\right)$, then the recurrence of each class $P_{i}\left(F_{i}\right)$ is its probability. Then the measure of the heterogeneity of the crystal forms, that is, the complexity of the snow layer (SL), is expressed as the quantity of coupled information $H(\mathrm{SL}) \equiv H_{n}\left(F_{n}\right)$ on the basis of K. Shannon's formula [Kustler, 1957]:

$$
H_{n}\left(F_{\mathrm{n}}\right)=-\sum_{i=1}^{n} P_{i} \cdot \log _{2} P_{i}
$$

where $P_{i}$ is the probability (encounter ability) of reiterative crystals of a given class of forms. The magnitude $H$ is the information negentropy and is expressed in binary units (bits). The complexity of the system also may be evaluated thermodynamically in the form of physical negentropy: $S=$ $k \cdot H$, where $k$ is the Boltzmann constant equal to $3.298 \cdot 10^{-24} \mathrm{cal} / \mathrm{deg}$.

\section{Result a of Empirical Modeling for Evolutionary Processes}

\subsection{Symmetry of Crystals and Its Conformity to Hydrothermal Fields in the Snow Pack}

The morphology of a crystal surface, emerging as the direct product of the interactions in the systems crystal-vapor and crystal-crystal, is capable of providing the diverse information about the surrounding medium that is practically impossible to derive even in a laboratory, to say nothing about the conditions of work in the field. The crystal-morphological analysis of snow structure may be employed as an effective method of interpreting the conditions and processes of internal transformations in the snow cover.

The surface faceting of growing depth hoar crystals is compromising in character because the medium "attaches" to their form a symmetry not natural to the internal structure of ice. There emerges an entire series of false (distorted, induced) forms of crystal growth, the symmetry of which is a subgroup of the true crystal symmetry of ice, that is, the plane-axial prism of symmetry of the hexagonal group, with the symmetry formula $\mathrm{L}_{6} 6 \mathrm{~L}_{2} 6 \mathrm{PaPeC}-6 / \mathrm{mmm}$ (see Foot-note to Table 1 and Fig. 2, a, b) . Such a crystal may grow under conditions of a maximally uniform medium with the symmetry of a sphere and stationary cylinder (Table 1 ; Fig. 1,a). 

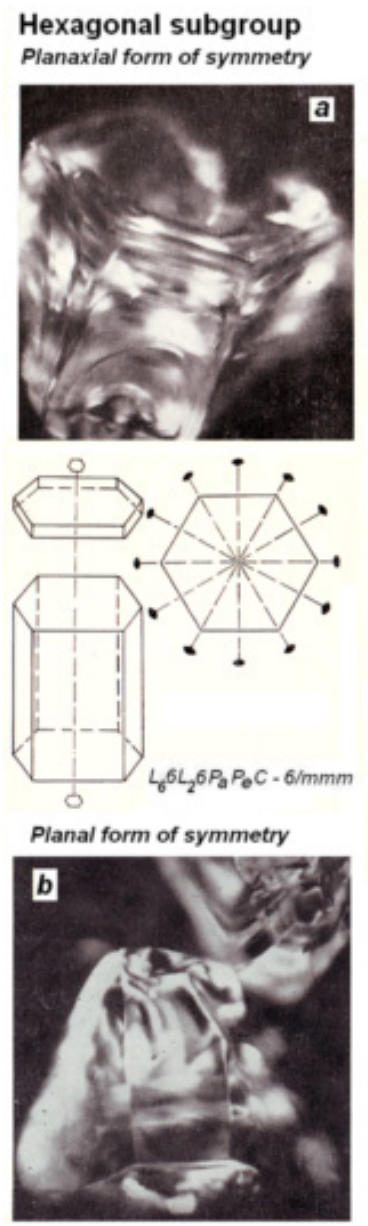

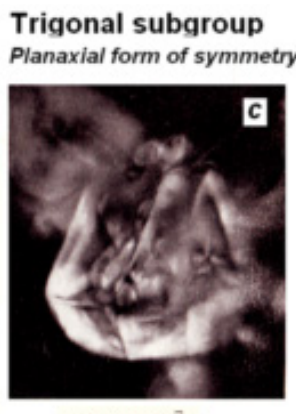

$\iota_{3} 3 l_{2} 3$ PPID $-3 \vec{m}$
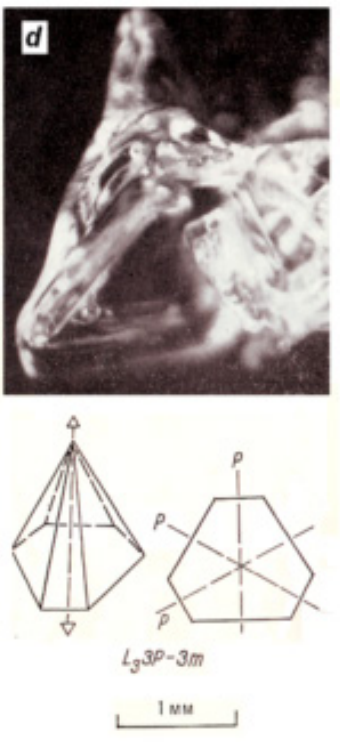

Rhombic subgroup
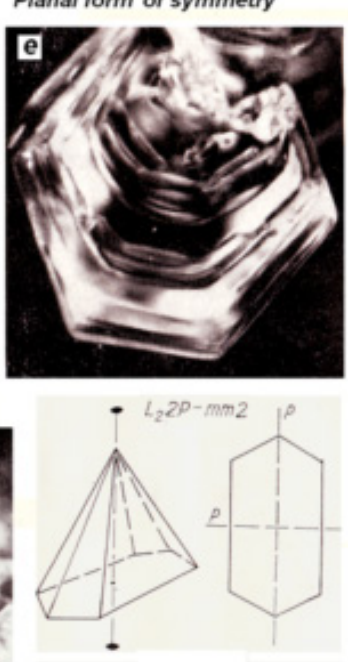

Triclinic subgroup Center form of symmetry

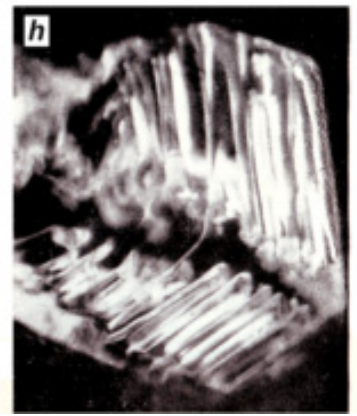

Monoclinic subgroup

Planaxial form of symmetry
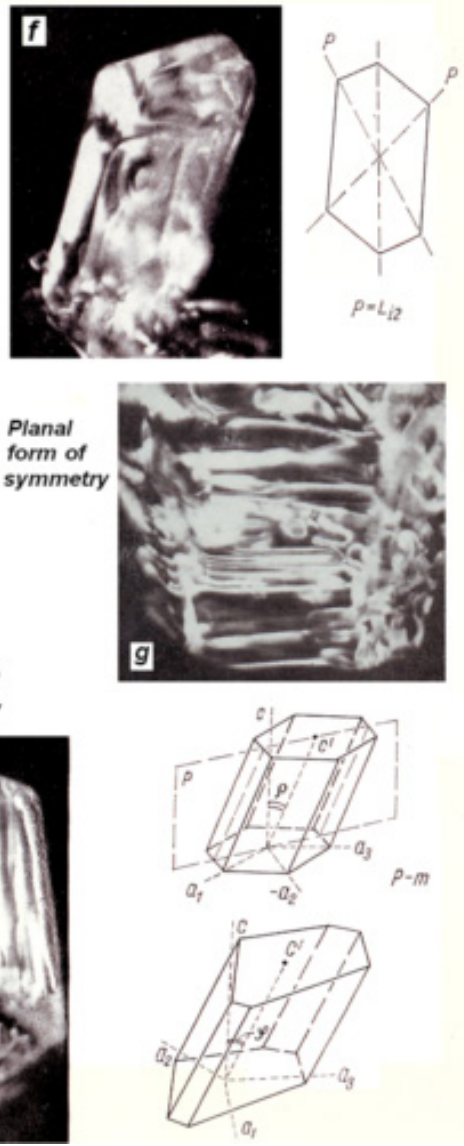

Figure. 1. Geometric symmetry of the crystals of sublimation metamorphism. Explanation in the text.

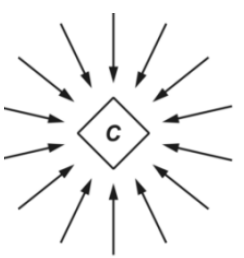

a

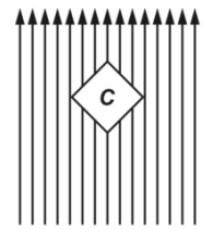

d

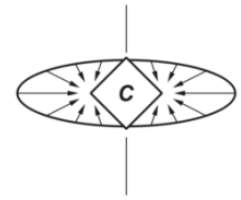

b

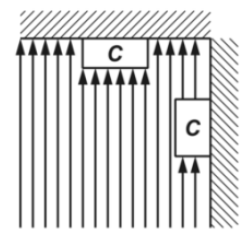

e

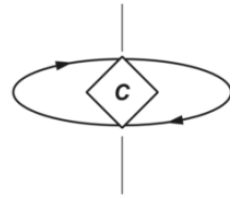

c

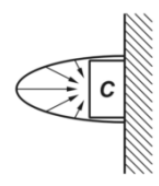

$\boldsymbol{f}$
C - growing crystal (arrows indicate the direction of fluxes of feed into the crystal); a - sphere symmetry; b, c - symmetry of stationary and rotating cylinders, respectively; $d$ - symmetry of a stationary cone; $\mathrm{e}$ - growth of crystals attached to a wall in a unidirectional medium with cone symmetry; $\mathbf{f}$ - same in a medium with cylinder symmetry.

Figure 2. Simple types of symmetry of crystal-forming mediums in the snow cover.

The hydrothermal field of the snow pack and the field of relaxation of its mechanical stresses have a lower symmetry
(Fig. 2, $d, e$ ). These fields have the property of polarity ("sign"): usually they lack a horizontal plane of symmetry, and the number of vertical planes is sharply reduced. With the same parameters of macrosymmetry of fields in the snowpack, the external symmetry of growing crystals is highly dependent on their orientation and location relative to each other. The less the elements of symmetry of the crystal and medium coincide, the lower the geometric symmetry of the resulting form. According to the statistical law of Fedorov-Grot [Grigoryev, Zhabin, 1975; Shubnilov, 1975], the more complex the composition of the medium, the lower the symmetry of the crystal form.

The forms of the trigonal subgroup point to the polarity of the hydrothermal field in the snow on the horizontal. The pyramidal outlines of the hexagonal and ditrigonal prisms are evidence that there is polarity of the medium on the vertical. This points to frequent but rhythmically recurring changes in the temperature and in the parameters of vapor diffusion around the crystals. The trigonal-rhombohedral and trigonal-pyramidal forms characteristic of the initial stages in the development of depth hoar (Fig. 1, $c, d$; and see below Fig. $5, b, e, h)$ grow in mediums with the symmetries of rotating cylinders and cones (see Table 1and Fig. 3, $b, d$ ). 
Table 1. Guiding morphological features of depth hoar crystals for determining their symmetry

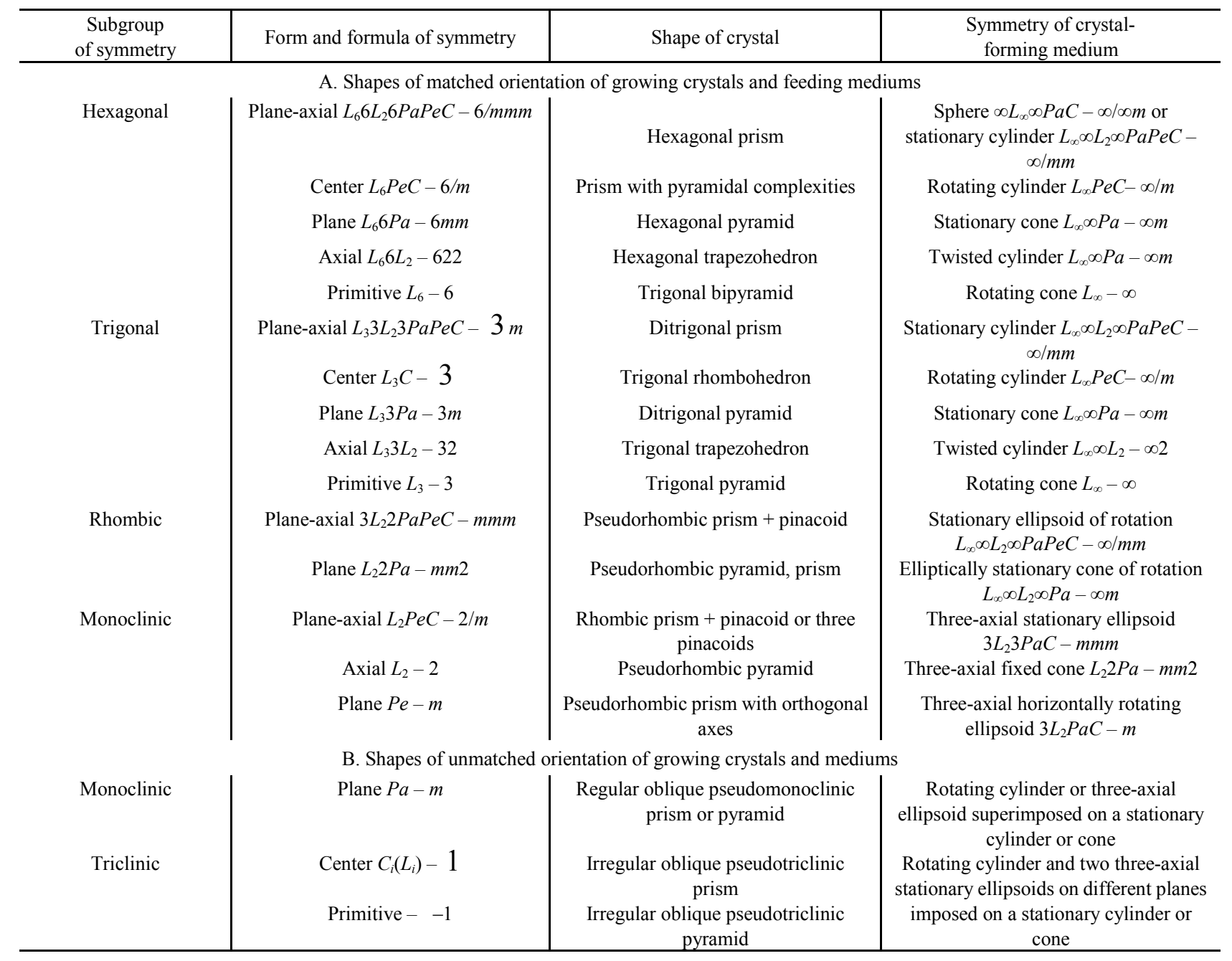

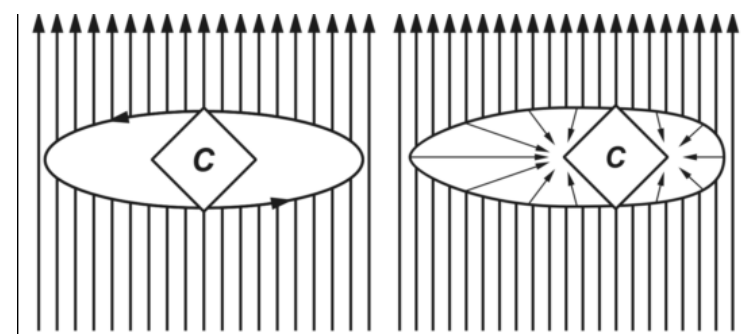

a

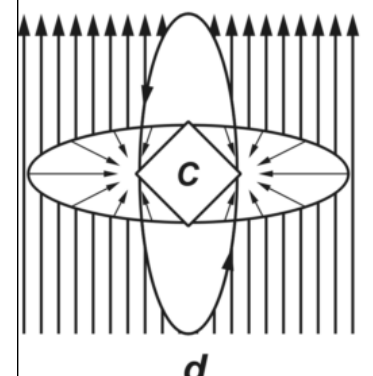

d

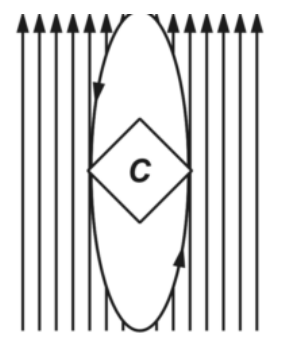

C

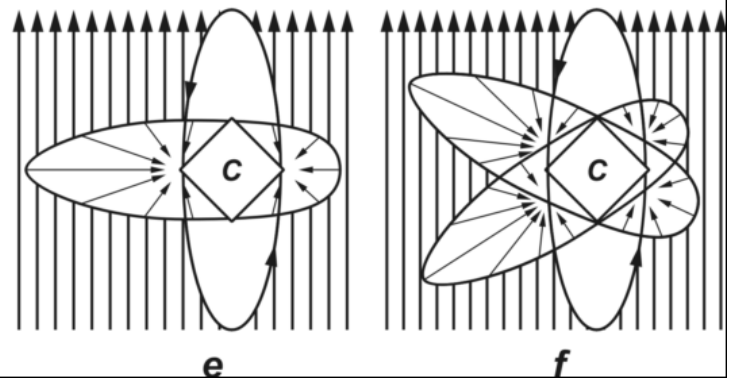

$\boldsymbol{a}, \boldsymbol{c}$ - combination of feeding fluxes with the symmetries of a cone and rotating cylinder; $\boldsymbol{b}$ - combination of fluxes with symmetries of a cone and stationary ellipsoid of rotation; $\mathbf{d}, \mathbf{e}, \mathbf{f}$ - various combinations of the symmetries of a feeding medium - cone, stationary and rotating cylinder and stationary ellipsoid of rotation. Other nomenclature as in Fig. 2.

Figure 3. Composite types of symmetry of crystal-forming mediums in the snow cover. 
The crystals of all remaining subgroups belong to the false forms of the hexagonal branch. The pseudorhombic forms point to the existence in the horizontal plane of one or two mutually perpendicular directions along which the gradients in the medium undergo marked changes. The medium is described by the symmetry $\infty=/ \mathrm{mm}$ for the prism and $\infty \mathrm{m}$ for the pyramid. The pseudorhombic forms usually dominate amid forms of depth hoar.

When gradients of force fields change even more abruptly and frequently, development takes place of pseudomonclinic and pseudotriclinic forms - typical representatives of "mature" depth hoar (see Fig. 1, $g, h$; and below Fig. 5, $f, g, j$, $k$ ). A characteristic of prisms $2 / \mathbf{m}$ is a medium with a "brick" symmetry - a three-axial stationary ellipsoid (see Table 1; Fig. $1, g$ and $3, b$ ). Beveled pseudomonoclinic single crystals just as all forms of the triclinic subgroup, have the lowest geometric symmetry in view of the almost non-matching of elements of symmetry of the crystal and the medium. This is due to both the non-steady state of the hydrothermal field and the contact interaction of the crystals themselves.

The growth conditions of planar and beveled pseudomonoclinic forms may be described in first approximation as the symmetry of a rotating cylinder superimposed on the flow of macroscopic diffusion in such a way that the vector of this flow is located on the plane of the rotating cylinder (see Fig. 3, c). Similar conditions may occur during sharp and frequent temperature fluctuations in. the snow layer (these fluctuations geometrically signify a change of "sign" of rotation of the cylinder). As a result, the crystal is forced to expand on the rotation axis of the cylinder, that is, in a general case horizontally.

Similar compression of form is also caused by the recrystallization according to the Rikke principle [Grigoryev, 1965; Grigoryev, Zhabin, 1975; - in conditions of one-sided pressure (compression). If the field of local diffusion or local stresses in the horizontal plane is described additionally by the symmetry of a stationary cylinder (see Fig. 3, d), then there develop oblique or planar, but regular in their base, hexagonal forms. When the horizontal symmetry of a three-axial stationary ellipsoid is present in the local medium (see Fig. 3, e) compressed and beveled pseudomonoclinic prisms and pyramids form with a single vertical plane of symmetry.

Finally, mediums with the most complex shape of symmetry (see Fig., 3, $f$ ) are characteristic of pseudotriclinic forms. At least three locally rotating flows are superimposed on a unidirectional macroforce field. One of them with the symmetry of a rotating cylinder causes the form to compress. The two others move on planes located at an angle to the vector of the macroforce field, and they have the symmetry of a three-axial stationary ellipsoid. This gives the crystal form a "triclinic" outline (see Fig. 1, $h$, and below Fig. 5, $g, j$, $k)$.

As is known, layers with well-developed depth hoar often have a well-defined vertical columnar (fibrous) texture. The fibers are composed of not only relatively symmetrical columnar crystals but also planar single crystals with reduced symmetry. The latter often develop on the walls of air pores. The higher symmetry of the crystals points to those situations when the pores are fairly evenly distributed in the three-dimensional space of the snow layer and, consequently, the areas of the contacts between particles are of one order both on the layers and in the vertical cross section. On the other hand, the well-defined dissymmetry of the forms, particularly the planar types, is an indication that there is a prevalence of vertical pores and accretions (clasters) weakly connected to each over the extent of the snow stratum. It is evident that in the second case the layer is more brittle to sublayer shear and hence more avalanche-hazardous (with the same specific area of contacts). A reduction in the symmetry of growing crystals in its mass is an indication that a dangerous columnar texture has developed in the layer.

\subsection{Deterministic Model of Sublimation Snow Metamorphism}

In order to determine the degree of order in the effect of different natural factors on change in the crystal-morphology of snow based on data from fixed observations in the central taiga of West Siberia, the author carried out dispersion analysis on variations in the dimension of an averaged crystal and negentropy using a known computational technique. He examined a three-factor set which took into account the following effects: $A$ - the character of the location of the snow profile, that is, the type of landscape facies, $B$ - the heights above the ground surface and $C$ - the growth of a given layer of snow. Results of the computation showed (Tables 2 and 3) that the principal factor in the change of snow structure is time, that is, layer growth. The time factor determines the highest degree to which crystal forms and dimensions vary. Stage growth of crystals depends by $45-60 \%$ on the age of the layer. The height of the layer above ground also has a substantial effect. This factor only slightly lags the growth factor in importance and predetermines the secondary differentiation of the snow profile [Kolomyts, 1976]. Local site has the least effect on the variability of the crystals; much more substantial is the effect of the combined factors of layer age and height. Local differences in the structure of the snow pack are disclosed only through comparing the results of simultaneous (within several days) measurements in different local sites and certainly with account taken of layer height. The effect of the above factors is a function of the continentality of the winter. In general, the higher the continentality of winter conditions, the more effective the time factor and the weaker the spatial differences in the construction of the snowpack. 
Table 2. Results of dispersion analysis of variation in the radius and volume of an averaged crystal in the snow cover of the Konda-Sosva-Ob river region (West Siberia)

\begin{tabular}{|c|c|c|c|c|c|c|c|c|c|c|}
\hline \multirow[t]{2}{*}{ Winter } & \multirow[t]{2}{*}{ Parameter* } & \multicolumn{9}{|c|}{ Factors and their combinations** } \\
\hline & & $A$ & $B$ & $C$ & $A B$ & $A C$ & $B C$ & $A B C$ & $Z$ & $X$ \\
\hline \multirow[t]{3}{*}{$1965 / 66$} & $S$ & 1.1 & 34.9 & 45.9 & 7.9 & 2.1 & 1.9 & 6.2 & 0 & 100 \\
\hline & $v$ & 4 & 4 & 3 & 16 & 12 & 12 & 48 & 0 & 99 \\
\hline & $\sigma^{2}$ & 4.5 & 138.9 & 245.0 & 7.9 & 2.8 & 2.5 & 2.8 & 0 & 16.2 \\
\hline \multirow[t]{3}{*}{$1967 / 68$} & $S$ & 4.2 & 35.8 & 43.1 & 6.9 & 1.3 & 3.2 & 5.5 & 0 & 100 \\
\hline & $v$ & 2 & 4 & 3 & 8 & 6 & 12 & 24 & 0 & 59 \\
\hline & $\sigma^{2}$ & 16.0 & 68.7 & 110.3 & 6.6 & 1.7 & 2.1 & 1.8 & 0 & 13 \\
\hline \multirow[t]{3}{*}{$1968 / 69$} & $S$ & 0.6 & 16.2 & 45.7 & 9.1 & 3.8 & 6.8 & 16.6 & 1.2 & 100 \\
\hline & $v$ & 4 & 4 & 3 & 16 & 12 & 12 & 48 & 0 & 99 \\
\hline & $\sigma^{2}$ & 1.5 & 44.5 & 168.0 & 6.3 & 3.5 & 6.2 & 3.8 & 0 & 11.1 \\
\hline
\end{tabular}

$* S$ - magnitude of parameter variability as a function of the different factors (\%); $v$ - number of degrees of freedom; $\sigma^{2}-$ degree of variation (dispersion).

** $Z$ - unaccounted (random) factors; $X$ - total effect of all factors and their combinations; for remaining symbols see the text.

Table 3. Results of dispersion analysis of variation in the crystallomorphological complexity (negentropy) of snowpack layers (Kondo-Sosva-Ob river region)*

\begin{tabular}{|c|c|c|c|c|c|c|c|c|c|c|}
\hline \multirow{2}{*}{ Winter } & \multirow{2}{*}{ Parameter } & \multicolumn{9}{|c|}{ Factors and their combinations } \\
\hline & & $A$ & $B$ & $C$ & $A B$ & $A C$ & $B C$ & $A B C$ & $Z$ & $X$ \\
\hline \multirow{3}{*}{$1967 / 68$} & $\mathrm{~S}$ & 5.5 & 33.4 & 38.2 & 4.4 & 6.6 & 5.9 & 5.9 & 0 & 100 \\
\hline & $v$ & 2 & 4 & 3 & 8 & 6 & 12 & 24 & 0 & 59 \\
\hline & $\sigma^{2}$ & 7.5 & 22.5 & 34.3 & 1.5 & 3.0 & 1.3 & 0.7 & 0 & 4.6 \\
\hline \multirow{3}{*}{$1968 / 69$} & $\mathrm{~S}$ & 3.8 & 11.2 & 58.3 & 4.9 & 2.9 & 3.4 & 9.7 & 5.8 & 100 \\
\hline & $v$ & 4 & 4 & 3 & 16 & 12 & 12 & 48 & 0 & 99 \\
\hline & $\sigma^{2}$ & 9.2 & 27.5 & 190.7 & 3.0 & 2.3 & 2.8 & 2.0 & 0 & 9.8 \\
\hline
\end{tabular}

* Symbols are as in Table 2.

Later on low-mountain Regions of the Lower By-Amur river it has been revealed the next dependence percentage portion of skeleton crystal shapes $N_{\text {sk }}$ (as a culmination of metamorphism, see below) from the age of given genetic snow layer $\tau$ :

$$
N_{\text {sk }}=0.3136 \cdot \tau-545 ; \quad \mathrm{R}=0.728 ; \quad \mathrm{R}^{2}=0.530 .
$$

There $\mathrm{R}$ and $\mathrm{R}^{2}$ - accordingly the coefficients of correlation and determination. The height above the ground of the latter is exhibited much weaker (by 15-35\%). The local site of snow pack has the minimum effect $(0.5-5.5 \%)$ on crystal growth. Time makes snow recrystallization processes irreversible and causes translational motion in crystal growth and in "alteration of generations" of crystal forms, in the alternation of periods of their development and degradation. The main evolutionary unit of snow cover is a genetically integral snow layer as an elementary self-developing natural community of crystalline individuals of different shape.

The author has developed an empirical deterministic model describing the unclosed evolutionary sublimation-metamorphic cycle of seasonal snow cover and regional (polymorphic) versions of this cycle (Fig. 4). The superposition of natural symmetries in the snowpack acts as a specific process distended in time. The medium (the diffusion field of water vapor) imposes its symmetry on the growing crystal with some graduation, with staged changeover in the mechanisms of interaction in the systems crystal-vapor and crystal-crystal. Therefore, "...any crystalline individual before becoming what is at the present time has passed through known phases of growth" [Shubnikov, 1975, p. 82].

Dry snow cover during the course of winter tends to traverse the directional trajectory of the sublimation-metamorphic cycle consisting of three periods: destructive (I), constructive (II) and regressive (III). These periods include nine stages of growth and subsequent decomposition of the crystals (see Fig. 4): the fragmentation stage $\left(\mathrm{I}_{1}\right)$, polyhedral $\left(\mathrm{I}_{2}\right)$, stages of planar and columnar faceted prisms $\left(\mathrm{II}_{1}\right.$ and $\left.\mathrm{II}_{2}\right)$, semiskeletal and skeletal stages $\left(\mathrm{II}_{3}\right.$ and $\left.\mathrm{II}_{4}\right)$, sectorial $\left(\mathrm{III}_{1}\right)$, plate-like $\left(\mathrm{III}_{2}\right)$ and, finally, the sublimation-firn stage ( $\left.\mathrm{III}_{3}\right)$. Crystals in each growth stage produce corresponding classes of forms with the same names. Each layer of the snowpack passes through similar stages of evolution, designated as phases of metamorphism. All stage forms of constructive metamorphism belong to category of depth hoar, in broad interpretation of this notion.

Destructive snow metamorphism (decomposition of primary crystals and their transformation into shapeless "grains") is effective only under the weak gradient thermal field of snow pack, therefore it is the longest in the regions with soft (oceanic) winters. In strongly continental winter conditions, the "grainy" (polyhedral) stage actually drops out, while the fragmentation stage becomes shorter. 

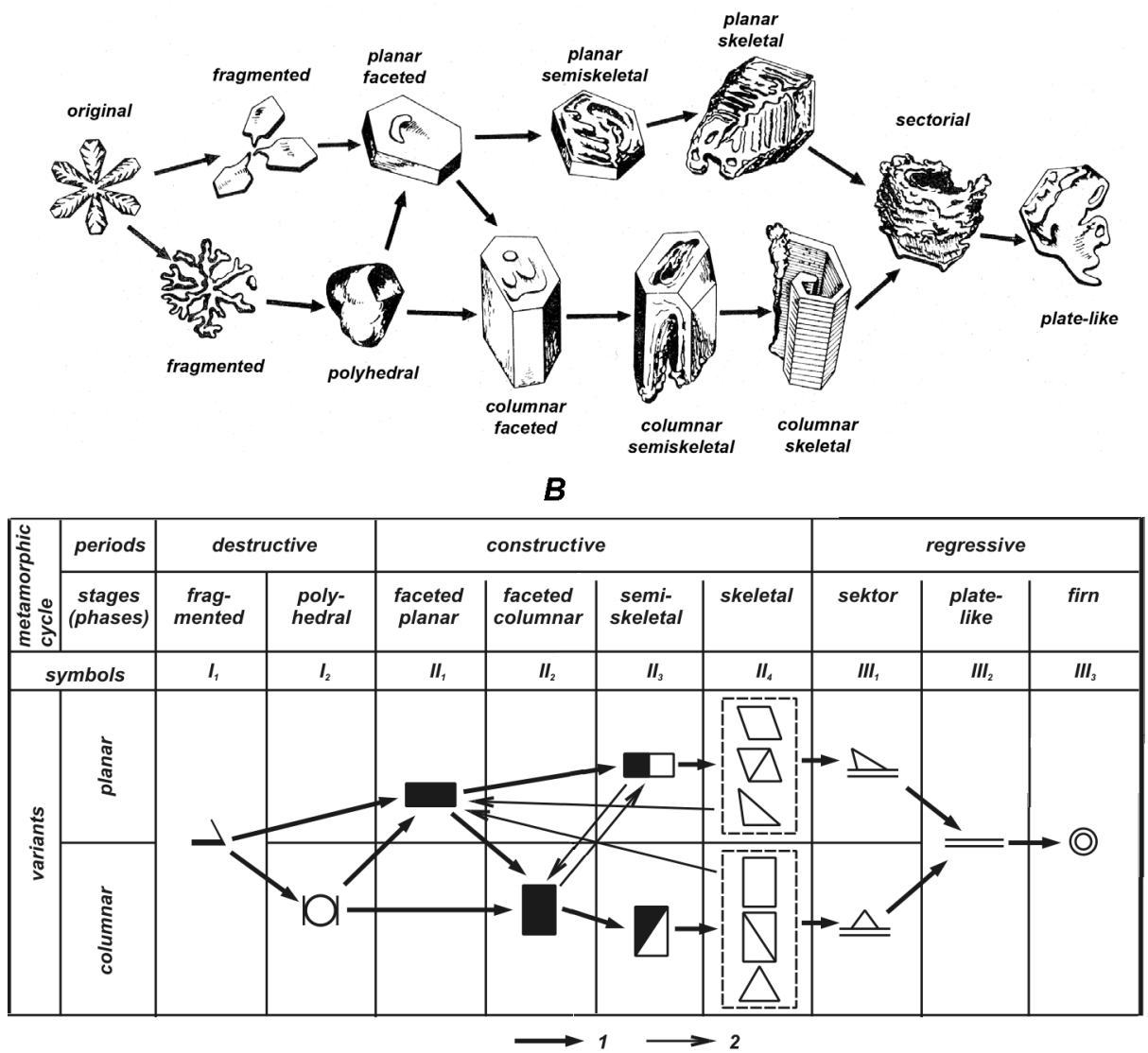

$\boldsymbol{A}$ - the main milestones of crystal ontogenesis in dry snow cover, their individual stage growth and following decomposition. Shown are two main variants (programs) of sublimation ontogenesis are showed : planar (above) and columnar (below).

$\boldsymbol{B}$ - principle scheme of sublimation-metamorphic cycle. $\quad 1$ - transitions of the basic (elemental) chain of transformations of crystal forms and snow layers; 2 - transitions of age complexity in the structure of layers and their shift from one variant (program) of metamorphism to another.

Figure 4. The sublimation-metamorphic cycle of dry seasonal snow.

Constructive metamorphism. Stages in the growth of planar and columnar faceted prisms and pyramids Faceted planar prism
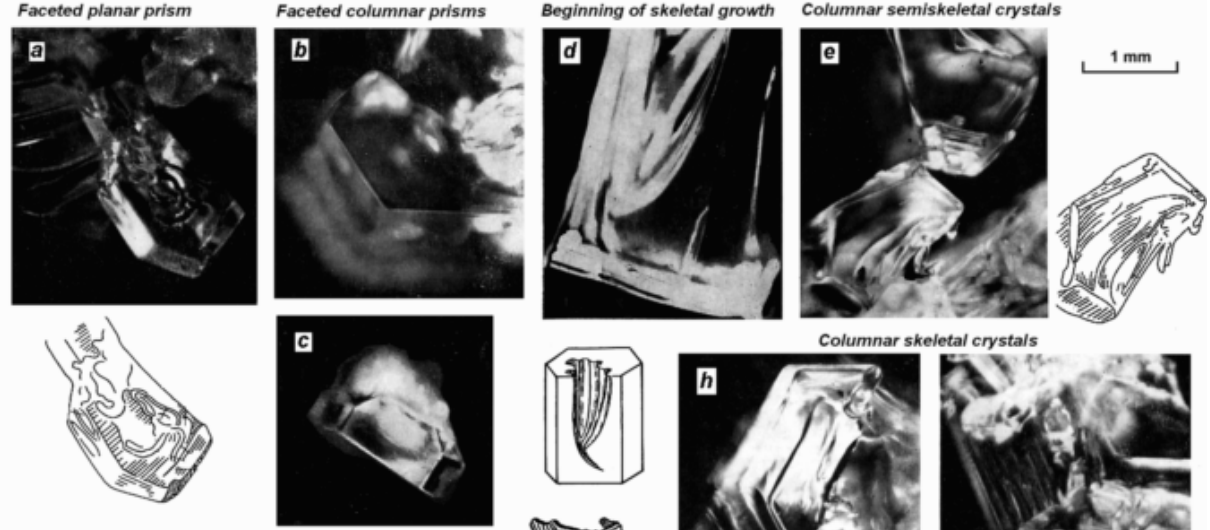

Stages in the skeletal growth of crystals
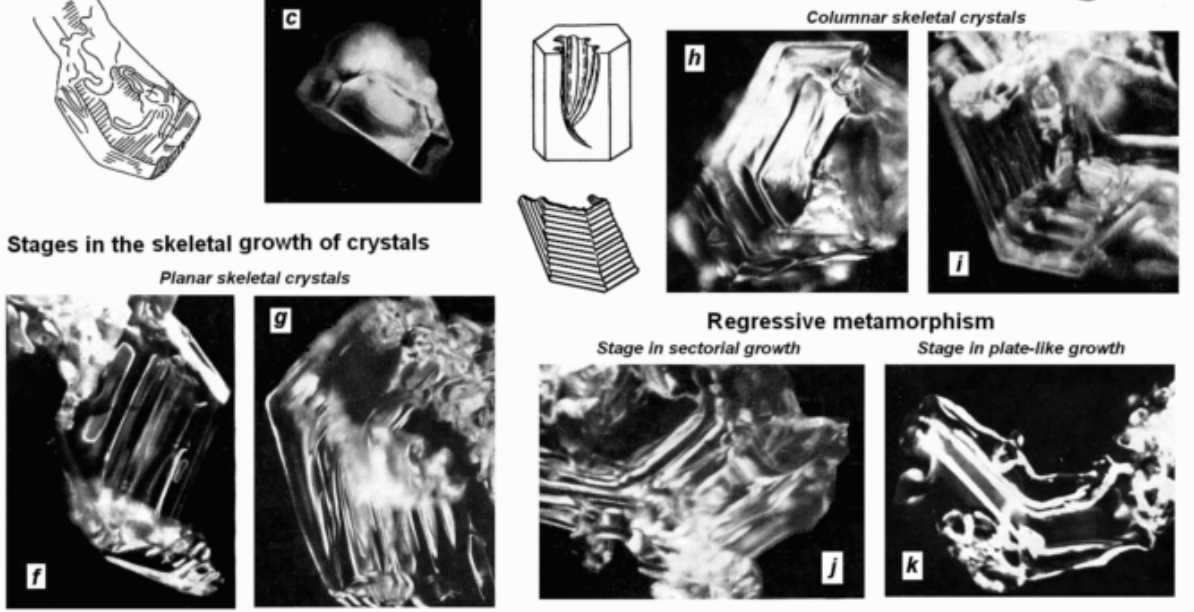

Figure 5. Stage shapes of the growth and decomposition of depth hoar crystals in loose and weekly compacted snow. Explanation in the text. 
The degree of snow re crystallization is determined by the efficiency of constructive metamorphism (see Fig. 4, B), which takes the most part of winter in continental regions. Variation and statistical analysis has shown [Shubnikov, 1975; Kolomyts, 1977], that crystal-morphological complication of the layer is conditioned by selection of crystals during their growth. The groups of particles are converted from one crystalline form into another not simultaneously; therefore, the layer is gradually enriched in various classes of crystal forms.

A particular crystalline shape corresponds to each stage in the growth of a monocrystal. The succession of stages is accompanied by regular changes in its dimensions (radius, volume); at first the dimensions increase comparatively slowly (until the skeletal stage) and then they decrease more rapidly (Fig. 6). Within each shape (stage of development) there is metric (linear and volumetric) growth of the crystal, the transition from one shape to another (stage succession) signifying its structural growth. A generation of crystal shapes in its familiar meaning is similar to the concept of "species generation" in biology. The formation of a new crystalline form from an old one is a qualitative jump in the process of the continuous metric growth of the crystal, a discontinuity in the course of this growth, a sort of "burst" resolving the contradictions which occurred in the preceding stage of growth.

In the beginning of constructive metamorphism, crystals comprise comparatively homogenous classes of forms subject to the laws of normal (Gaussian) distribution. At the stages of faceted growth, crystals are yet of comparatively small sizes, therefore the tendency to simplicity and perfection of the form is predominant, according to the Gibbs-Curie-Wulff principle [22, 53]. Solid crystal forms of faceted classes are for the most part highly symmetrical (hexagonal, trigonal and rhombic), with repeatedly growing smooth facets (Fig. 5, $a, b, c$; see also Fig. 1, b, c). They are a morphological manifestation of the initial correct growth of crystals.

Faceted growth is accompanied by plastic-viscous deformations of snow ice crystal lattice resulting in its slow setting and packing. The duration of faceted stages and phases abruptly increases and the rate of crystal growth decreases in the direction from lower to upper snow layers.

Later on when the crystals reached the certain critical sizes they are transformed first into semi-skeletal forms, with appearance of "multi-sloped caps" and development of caverns (see Fig. 5, $d, e$ ). After that these forms turn into own skeletal crystals - openwork, hollow, with large striated facets (see Fig. 1, $d$, e, and Fig. 5, $h, i$ ). Such morphological complication implies gradual inclusion of the medium into a crystal (in accordance with the principle of superposition of symmetries) and development of adaptation mechanisms providing its further growth [46]. This is the first qualitative leap in the metamorphic cycle. The adaptive tendency of particle growth (by the principle of maximum rate of crystallization completion) becomes predominant, giving great diversity of skeletal forms. The rate of metamorphism drastically increases, however there is an abrupt decrease in the real symmetry of "forced" forms, up to the monoclinic plane and triclinic primitive symmetry (see Fig. $1, g, h$, and Fig. 5, $f, g$ ).

Skeletal rib shapes are the largest and the most complex morphological formations in snowpack. The large skeletal shapes of deep hoarfrost signify the highest stage of adaptive tendency of monocrystal growth and, simultaneously, the final stage of constructive metamorphism. At this stage, the environment completely "imposes" an unusual shape on the growing crystal, uncoupling separate units of its internal molecular colony; however, the individual still preserves its integrity as a genetically whole formation. Skeletal crystal shapes are nothing but the most "mature" deep hoarfrost, creating the "weak layers" in snow pack: the threatening indication of a potential impending snow avalanche on avalanche-hazardous mountain slopes.

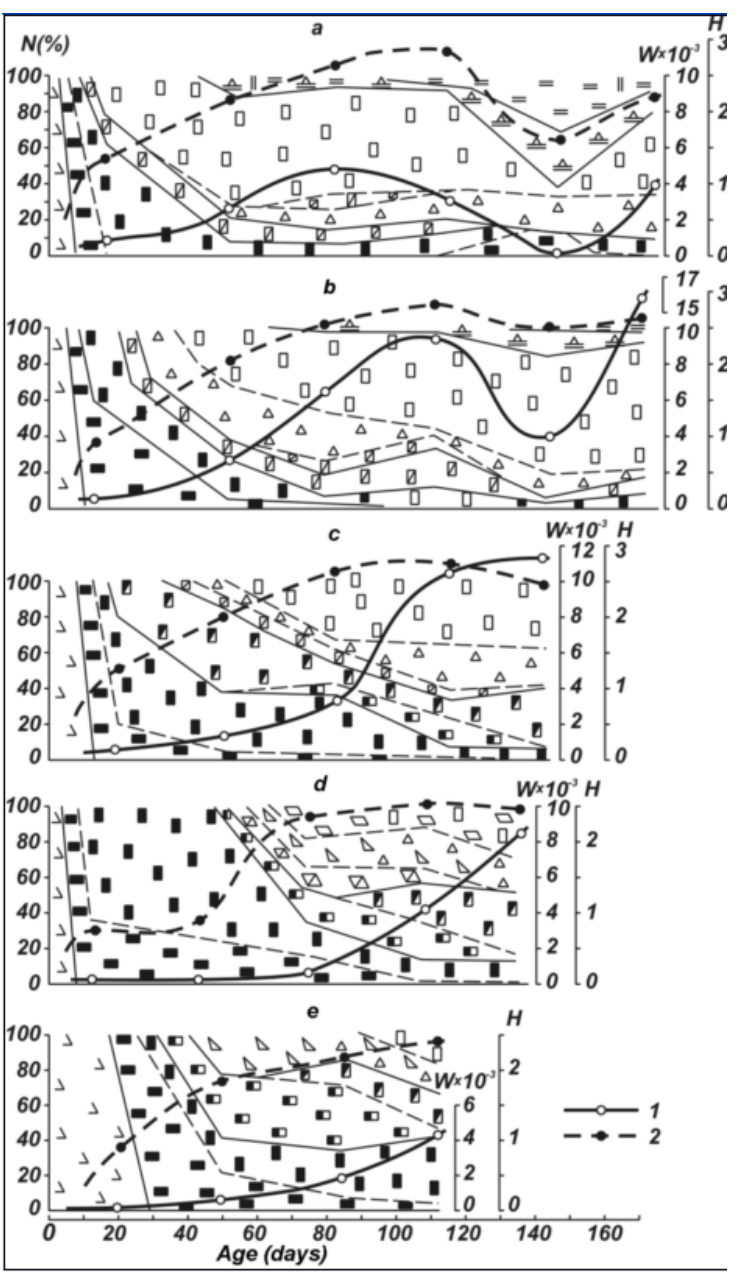

Parameters: $N$-portions of different classes and types of crystal forms (by there summary cubic capacity); 1 - average cubic capacity of crystal $\left(W \cdot 10^{-3}\right.$, $\left.\mathrm{cm}^{3}\right) ; 2$ - the information measure of structural diversity (negentropy) of layers $(H$, bytes); see formula (1). Height of the snow layer over soil surface, cm: $a-0 ; b-10 ; c-30 ; d-40 ; e-60$. The symbols of crystal forms see on Figure 4, $B$, and in Table 6.

Figure 6. The age changes of structure parameters of snowpack layers in taiga-swamped nature complex (middle taiga of West-Siberian Plain, winter of years 1968/69).

The complication of shape composition increases the 
negentropy (the measure of layer complexity) from 0 in the beginning of constructive metamorphism to 2.5-3 bit during the maximum development of skeletal crystals (see Fig. 6). Consequently, the skeletal phase is the highest degree of sublimation-metamorphic transformations of snow layer.

Because heterogeneous crystals grow much more rapidly than homogeneous ones [Sheftal, 1973], the proportion of faceted forms decreases sharply with the development of skeletal individuals (see Fig. 6). The constructive metamorphism of snow involves the mechanisms of natural selection of growing crystal individuals and, by analogy with the evolution of biological systems [Shmalgausen, 1968; Yablokov, Yusufov, 1976], this selection may be considered the principal motive force of directed evolution of crystal communities in snow layers. The age-related increase in shape diversity of the community is determined by the factor of "evolutionary time" [Pianka, 1978]; therefore, "... knowing the direction of selection, studying the regularities of evolution ..., we can predict with some degree of accuracy the direction of evolution of a given group" [Timofeev-Resovsky et al., 1977, p. 199].

Regressive metamorphism is the backward "movement" of crystalline individuals and snow layers: in the direction of simplification of their structure and approach to the state with the maximum entropy. This is the second qualitative leap in the metamorphic cycle. It manifests itself in the splitting and breakup of crystals under relatively unchanged external conditions [Sheftal, Kolomyts, 1973], hence it may be called snow "ageing", in the true sense of this notion. That takes place as a rule in the lower layers of snow lying on waterlogged ground that promotes vigorous migration of water vapors and rapid, growth of crystals. The large pressure of snow rising above these layers causes the regressive crystal changes as well.

"Evolutionary adaptation, of course, must have certain limits; ...ontogenetic potentials of this group of organisms are always limited" [Timofeev-Resovsky et al., 1977, p. 159]. In snow cover, such limit is signified by replacement of constructive metamorphism with regressive one. Further transition to the sectorial and lamellar stages of regressive metamorphism implies that the crystal first "decrepitates", being covered with small sectorial outgrowths (see Fig. 5, $j$ ) and then falls to pieces and acquires a kind of imperfect shape, becomes asymmetrical, with many internal defects, inclusion in the environment.

In this period the adapting of the growing crystal to the surrounding medium reaches its limit and appears in insoluble conflict with its internal tendency toward perfection of form. In the process of skeletal growth the medium is so embedded in the crystal's makeup and so actively "in charge of" (in the words of [Sheftal, Kolomyts, 1973]) its growth that the crystal in the end loses its individuality as a whole form. On the other hand, the morphological intricacy of a crystal in the skeletal stage of growth attains a level such that the asymmetry of its form substantially exceeds the asymmetry of the feed medium. This progressive "excess organization" of the crystal form also leads to a disruption of its stability [Shmalgausen, 1969]. The crystal begins to degenerate and then break up into separate parts - plate-like grains. Thin cloudy platelets, products of the splitting and disintegration of the monocrystals, have irregular outlines and also may be classified among the monoclinic-triclinic false forms. "In these final forms there are no traces of the approximate macrosymmetry, the traces being completely overcome by the asymmetry of the dominant unordered medium" [Sheftal, Kolomyts, 1973, p. 350]. During the sectorial and plate-like stages of this period (see Fig. 5, $j, k$ ), the sizes of particles abruptly decrease and many skeletal and faceted forms disappear (see Fig. 6).

The growth of crystals in the semi-skeletal, skeletal and sectorial stages can proceed in accordance with two variants (see Fig. 4): columnar $\left(\mathrm{II}_{3}{ }^{\mathrm{col}} \rightarrow \mathrm{II}_{4}{ }^{\mathrm{col}} \rightarrow \mathrm{W}_{1}{ }^{\mathrm{col}}\right.$ ) and planar $\left(\mathrm{II}_{3}{ }^{\mathrm{pl}} \rightarrow \mathrm{II}_{4}{ }^{\mathrm{pl}} \rightarrow \mathrm{W}_{1}{ }^{\mathrm{pl}}\right)$. These variants provide two types of crystal forms in each of the mentioned classes. Any of the variants of growth can dominate in the snow layer or in combination with the other provide a mixed variant. The variants of crystal growth may be regarded as distinct programs of constructive metamorphism. The polymorphic modifications of semisceletal and skeletal class shapes develop according to each of such program. These programs are "assigned" to the snow cover by meteorological conditions and primarily by the heat processes of winter.

Variants (or branches) of the sublimation-metamorphic cycle (columnar, planar, mixed) are predetermined on the one hand by the temperature state of the snow and, on the other, by the action of the force of gravity whose structural effect is dependent on the depth of deposition of the given layer, density of the overlying layers and the time of the loading action, that is, the age of the layer. Accordingly, in the first case the variants are branches of the sublimation thermal metamorphism of snow. The pressure factor displays only in the middle and lower layers of deep and dense depositions and causes of sublimation dynamic metamorphism. Columnar variant are predetermined under relatively high temperatures and faint temperature gradients (soft winter conditions), planar ones - under low temperatures and there sharp and frequent fluctuations (conditions of high continental winters). The field of snow compression is also of certain significance.

Variation and statistical analysis has shown that crystal-morphological complication of the layer is conditioned by selection of crystals during their growth. The groups of particles are converted from one crystalline form into another not simultaneously; therefore, the layer is gradually enriched in various classes of crystal forms, that is, the measure of its structural complexity increases (see Fig. 6), that is the entropy of system, by (Ashby, 1956), is reduces.

The evolutionary model of snow cover by no means envisages the passing by the sets "crystal form", "snow layer" and "snow pack" necessarily through all periods and stages (phases) of snow re-crystallization under any winter conditions. The point at issue is the invariant process of sublimation metamorphism as the major tendency of 
evolution of dry snow cover.

Such are the mechanisms of the stage transformations of crystal shapes in the process of the sublimation metamorphism of dry snow cover. The entire chain of such structural conversions may be visualized as essentially a single and generally well-defined open cycle analogous to the "life cycles" which are characteristic of the metamorphic bands of rocks in the lithosphere (Sutton, 1967). Thanks to the extraordinary dynamism of snow structure with time, over just a few months one can trace in it the same stages of metamorphism that in the earth's crust are stretched out over long geological periods. In this lies the general methodological value of using crystal-morphology to analyze snow structure.

\subsection{Evolutionary Character of the Sublimation-Metamorphic Cycle}

The sublimation-metamorphic cycle in its principal features is invariant and characterizes the process of snow cover self-development, which can be expressed by the known in ecology logistic (sigmoidal) curve [Ashby, 1956; Odum, 1983]. The trajectories of crystal sizes and measure of SL complexity have similar logistic-sigmoidal character (see Fig. 6), fully justifying the treatment of this type of structural transformation of the snow cover as an evolutionary process and the classification of the snow cover itself as a self-organizing natural system.

Irrespective of the conditions of location (zonal-sectorial on the plains, vertical differentiation in the mountains, landscape, topological, continentality of a given winter (within a certain range), snow density and height of the layer above the ground, each genetic group of crystals passes fully or partially through fundamentally the same stages of morphological changes, successively superseding each other. This process displays with bigger or lesser velocity under any temperatures, temperature gradients and intensity of vapor macroscopic diffusion (Table 4, Fig. 7). Passing through these stages is conditioned exclusively by internal interactions between growing crystals and dissymmetric hydrothermal fields in snow pack.

Table 4. Main hydro-thermal parameters of snow in different periods of winter 1968/69 and in presence of those or eithers phase states of snow layers (middlef taiga of West Sjberia)

$A$ Interfluve area with primary cedar-fir-fine grass-green/moss forest

\begin{tabular}{|c|c|c|c|c|c|c|c|c|c|c|}
\hline \multirow{2}{*}{$\begin{array}{l}\text { Period of } \\
\text { winter }\end{array}$} & \multicolumn{3}{|c|}{$\mathrm{H}=0 \mathrm{~cm}$} & \multicolumn{3}{|c|}{$\mathrm{H}=20 \mathrm{~cm}$} & \multicolumn{4}{|c|}{$\mathrm{H}=40 \mathrm{~cm}$} \\
\hline & $t$ & $d t / d h$ & $i \quad Q$ & $t$ & $d t / d h$ & $Q$ & $t$ & $d t / d h$ & $i$ & $Q$ \\
\hline $3 . \mathrm{XI}-7 . \mathrm{XII}$ & -5.4 & -03 & $2.2 \mathrm{II}_{2}^{\mathrm{col}}-\mathrm{II}_{3}^{\mathrm{col}}$ & -12.1 & -0.3 & $1.4 \mathrm{I}_{1}-\mathrm{II}_{2}^{\mathrm{col}}$ & - & - & - & - \\
\hline $7 . \mathrm{XI}-3 . \mathrm{I}$ & -5.1 & -03 & $2.0 \mathrm{II}_{4}^{\mathrm{Col}}$ & -11.3 & -0.3 & $1.4 \mathrm{II}_{2}^{\mathrm{Col}}-\mathrm{II}_{3}^{\mathrm{col}}$ & -78 & -0.4 & 1.1 & $I_{1}-I_{2}$ \\
\hline $3 . \mathrm{I}-8 . \mathrm{II}$ & -7.3 & -0.4 & $\mathrm{II}_{4}^{\mathrm{col}}$ & -14.0 & -0.3 & $13 \mathrm{II}_{3}^{\mathrm{col}}-\mathrm{II}_{4}^{\mathrm{col}}$ & -22.5 & -0.4 & 0.5 & $\mathrm{II}_{1}^{\mathrm{pl}}-\mathrm{II}_{3}^{\mathrm{pl}}$ \\
\hline $8 . \mathrm{II}-13 . \mathrm{II}$ & -5.8 & -02 & $\mathrm{II}_{4}^{\mathrm{Col}}$ & -99 & -0.2 & $09 \quad \mathrm{II}_{4}^{\mathrm{Col}}$ & -13.1 & -0.2 & 0.6 & $\mathrm{II}_{3}^{\mathrm{pl}}$ \\
\hline $13 \mathrm{III}-8 \mathrm{IV}$ & -3.1 & -0.1 & $\mathrm{III}_{1}^{\mathrm{col}}$ & -52 & -0.1 & $\mathrm{Ir}_{4}^{\mathrm{col}}$ & -70 & -0.1 & 0.2 & $\mathrm{II}_{3}^{\mathrm{pl}}-\mathrm{II}_{4}^{\mathrm{pl}}$ \\
\hline
\end{tabular}

$B$. Pine peatmoss forest swamp in interfluve depression

\begin{tabular}{|c|c|c|c|c|c|c|c|c|c|c|c|}
\hline \multirow{2}{*}{$\begin{array}{l}\text { Period of } \\
\text { winter }\end{array}$} & \multicolumn{3}{|c|}{$\mathrm{H}=0 \mathrm{~cm}$} & \multicolumn{4}{|c|}{$\mathrm{H}=30 \mathrm{~cm}$} & \multicolumn{4}{|c|}{$\mathrm{H}=50 \mathrm{~cm}$} \\
\hline & $t$ & $d t / d h$ & $Q$ & $t$ & $d t / d h$ & $i$ & $Q$ & $t$ & $d t / d h$ & $i$ & $Q$ \\
\hline $3 . \mathrm{XI}-8 \mathrm{XII}$ & -43 & -0.5 & $36 \mathrm{II}_{3}^{\mathrm{col}}-\mathrm{II}_{4}^{\mathrm{col}}$ & -166 & -0.4 & & $\mathrm{I}_{1}-\mathrm{II}_{\mathrm{lol}}$ & - & - & - & - \\
\hline $8 . \mathrm{XII}-5 . \mathrm{I}$ & -36 & -0.3 & $38 \mathrm{II}_{4}^{\mathrm{col}}$ & -9.9 & -02 & 1.4 & $\mathrm{II}_{1}=\mathrm{II}_{2}^{\mathrm{rol}}$ & - & - & - & - \\
\hline 5.I - 10.II & -49 & -0.4 & $3.7 \mathrm{II}_{4}^{\mathrm{col}}-\mathrm{III}_{1}^{\mathrm{col}}$ & -115 & -02 & & $\mathrm{II}_{2}^{\mathrm{Col}}-\mathrm{II}_{3}^{\mathrm{Col}}$ & -18.8 & -0.5 & 06 & $\mathrm{I}_{1}-\mathrm{II}_{1}$ \\
\hline $10 . I I-15 . I I I$ & -33 & -0.1 & $23 \quad \operatorname{III}_{1}^{\mathrm{sol}}$ & -7.5 & -0.1 & 09 & $\mathrm{II}_{4}^{\mathrm{col}}$ & -11.8 & -0.2 & 0.7 & $\mathrm{II}_{2}^{\mathrm{pl}}$ \\
\hline $15 \mathrm{II}-19 . \mathrm{IV}$ & -18 & -0.1 & $1.4 \mathrm{III}_{1}^{001}-\mathrm{III}_{2}$ & -3.7 & -0.1 & 0.7 & $\mathrm{II}_{4}^{\mathrm{Col}}$ & -5.7 & -0.1 & 05 & $\mathrm{II}_{3}^{\mathrm{pl}}$ \\
\hline
\end{tabular}

Footnote. H - height of layer under soil; $t$ - everage temperature, grad; $d t / d h$ - temperature gradient, $\mathrm{grad} / \mathrm{cm} ; i \cdot 10^{-3}$ - index of water steam diffusion, $\mathrm{g} / \mathrm{cm}^{2}$. day; $Q$ - phase state of snow layer (see Fig. 4.4,B). 

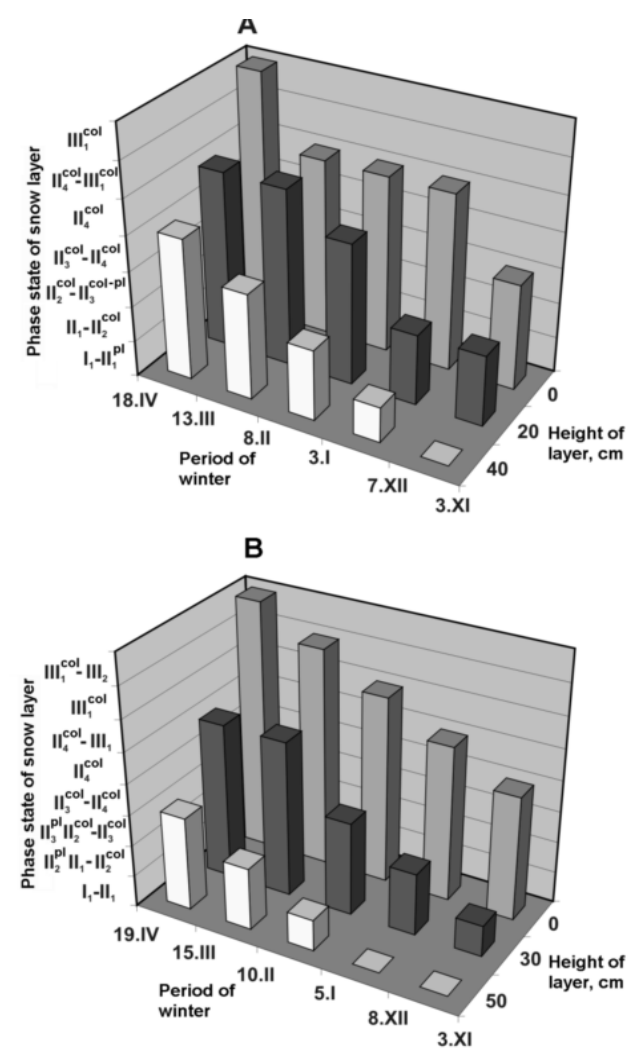

Local natural complexes: A - taiga-forest; $\mathrm{B}$ - taiga-bog.

Figure 7. Hystograms of seasonal changes in phase states of snow layers, being at different heights under soil and correspondingly in unindentical hydrothermal conditions (see Table 4)

Such findings result not only from the processes and phenomenon presented above. The evolutionary concept of the sublimation re-crystallization of dry snow cover being developed here is based on the following facts.

(1) the natural diversity of crystal forms present in one texturally homogeneous layer; this diversity substantially exceeds the diversity of the external (in relation to the snow cover) thermodynamic conditions;

(2) the distinct sequence in which one crystal form is transformed into another that invariably recurs each winter in different regions and in snow layers of different height [Kolomyts, 1976];

(3) the directional complication of the morphology of growing crystals with time, the progressive "divergence" of forms and the corresponding increase in the crystal-morphological complexity of each snow layer during the period of constructive metamorphism;

(4) the constant "fluidity" of visible forms of crystals, the presence in them of only relative stability in time; the inability of external hydrothermal conditions to rigidly and unambiguously determine the variability of crystal forms;

(5) the existence of many elements of morphological similarity between different crystal individuals; the stronger this similarity, the closer the visible forms in question are to each other in the time of their origination, indicating that the crystals have a mutual heritage with respect to their external morphology and that they can be fitted basically into one genetic set.

Solid crystal forms of faceted classes are for the most part highly symmetrical (hexagonal, trigonal and rhombic), with repeatedly growing smooth facets. They are a morphological manifestation of the initial correct growth of crystals, when particles have not yet reached the critical sizes [Honigman, 1958; Grigoryev, 1965]. Then they are transformed first into semi-skeletal shapes (with development of caverns) and further into skeletal crystals (openwork, hollow, with large striated facets). Such morphological complication implies gradual inclusion of the medium into a crystal (in accordance with the Curie's principle of superposition of symmetries) and development of adaptation mechanisms providing its further growth [Sheftal, 1973; Sheftal, Kolomyts, 1973]. Accordingly, there is an abrupt decrease in the real symmetry of these "forced" shapes, up to the monoclinic plane and triclinic primitive symmetry.

Invariance of the metamorphic cycle is expressed in stage change of the classes of crystalline shapes. The types of forms within each class characterize different variants of this cycle, which are determined by the external regulatory influence: the hydrothermal regime of the whole soil-snow-air system. External environment plays the role of an indicating mechanism switching over the metamorphic cycle trajectory to some or other development program. The major external factor is the degree of winter continentality, which determines the general level of snowpack cooling and nonstationarity of thermal field in its separate layers. The regime of snow accumulation determining the field of snow compression is also of certain significance.

The evolutionary model of snow cover by no means envisages the passing by the sets "crystal shape" (C), "snow layer" (SL) and "snow pack" (SP) necessarily through all periods and stages (phases) of snow recrystallization under any winter conditions. The point at issue is the invariant process of sublimation metamorphism as the major tendency of evolution of dry snow cover.

In terms of the systems theory, the structure of each genetic layer of snow pack is determined by diversity of crystal shapes and their various deviations from the most stable shape (some etalon state). Such etalon, as has already been shown, is the skeletal stage of their growth, when superposition of the symmetries of growing crystal and environment reaches its culmination. Deviation of the crystal community in snow layer from the etalon was expressed via the measure of its situational disordering. Disordering of the system is the measure of misalignment of state $i$ of each of its elements with its etalon state $x_{i \mathrm{et}}$. This measure tends to zero at $x_{i} \rightarrow x_{i e t}$. Since the etalon of orderliness is known beforehand, the trajectory of object's "movement" to its etalon may be considered as a program of its gradual changes (evolution). In this case, it will be the program of snow sublimation metamorphism. 
The variants of metamorphism differ in the boundaries of the etalon region and the optimums of orderliness. When winter is more continental, more crystals are involved in skeletal growth for the layer to reach the etalon region and the progressive transformations of snow structure (the period of constructive metamorphism) are longer. The optimum vector of layer ordering characterizes the "goal" of snow evolution mentioned above. When moving towards or away from this goal, the layer at each step has certain disordering relative to its optimum etalon. The parameters of such disordering (misalignment) will be the values of probability difference $P_{i j}-P_{\text {et } i}$ for each variable of the vector: the class of crystal shapes. In accordance with methodical developments [Pianka, 1978], the total disordering of snow layer $\bar{O}(\mathrm{SL})_{i}$ can be determined by the Euclidean distance:

$$
\bar{O}(S L)_{i}=\sqrt{\sum_{j=1}^{n}\left[\mu_{i j}\left(P_{i j}-P_{e t j}\right)\right]^{2}}
$$

where $P_{i j}$ is the probability (occurrence) of class $i$ of crystal shapes in layer $j, P_{\text {et } i}$ is the etalon value of probability of the same class at the optimum state of SL, and $\mu_{i j}$ is the weight factor characterizing the importance of this misalignment. This factor was obtained by calculations on the basis of implication relations between SL set elements.

It is most convenient to use the relative parameter of disorder expressed as a decimal fraction:

$$
P_{\bar{O}}=\bar{O}(\mathrm{SL})_{j} / \bar{O}(\mathrm{SL})_{\max } \text {. }
$$

The $\bar{O}(\mathrm{SL})_{\max }$ values were determined empirically as follows: $0.214,0.263$ and 0.336 for the columnar, mixed and planar variants of metamorphism, respectively.

\section{Crystal-morphological Classification for Snow on the Ground}

\subsection{The Overview of Existing Classifications}

None of the sciences has done without systematization of objects under study. Systematization is division of an entire set of objects into parts by the signs of similarity and their ranking by any sign so that the lowest rank would be referred to the highest one as a part to the whole. The first and second parts of systematization are called classification and taxonomy, respectively [Armand, 1975]. In the International glaciological literature, systematization of snow structure is usually referred to as "Classification for the snow of the ground". This term seems to be not quite correct. In our point of view, it would be more proper to term such classifications as "Guidance for description of seasonal snow cover".

Rather detailed chronology of development of foreign guidance on snow studies, from the middle of the $19^{\text {th }}$ century to 2000 , is presented in the work [9], which shows gradual transition from the merely descriptive method of structural analysis of snow sections to the texture-morphological method, with saturation of qualitative descriptions with metric and micromorphological characteristics, as well as the signs of the respective mechanical properties of snow. The advantage and practical value of such guides [Schaefer et al., 1954; Quervain de, 1958; Sommerfelfd and LaChapelle, 1970; Colbeck et al., 1990; Fierz et al., 2009] are that they reflect, as far as possible, all natural processes of snow cover formation and further changes: periodicity of snow accumulation, wind-blown snow stream and compaction of snow, part-melting and moistening of snow during thaws, with formation of ice and firn crusts, settling of snow, sublimation, regelation and re-crystallization processes of metamorphism in different layers of snow pack and, finally, the processes of firnification of snow resulting in its transformation into ice. During the field study of snow sections, these classifications may show the prevalent type of transformation of the structure and texture of different snow layers in the preceding period: sublimation, regelation, re-crystallization, etc. It means restoration of external conditions for internal transformation of snow cover.

\subsection{Construction of the Classification}

Crystal-morphological classification of snow worked out by the author may be attributed to Supplement A ("Grain shape classification") of last "International Classification for Seasonal Snow" [Fierz et al., 2009]. Our classification have been created on the basis of conformity to natural laws of the ontogeny of crystals in a snow pack is constructed on the genetic principle: all categories of the forms of individual crystals correspond to certain periods and stages of the sublimation-metamorphic snow cycle. The following 17 major specific crystalline forms revealed in dry snow cover were taken for taxonomic analysis: 1, 2, skeleton apical planar and spatial (dendrites); 3, isometric polyhedral; 4, 5, faceted prismatic planar and columnar; 6 , faceted pyramidal-prismatic ("bullets"); 7, 8, semiskeleton planar and columnar; skeleton rib-like prismatic and pyramidal forms: 9, 10, planar; 11-13, columnar; 14, skeleton rib-like pyramidal-prismatic ("cups" and "wineglasses"); 15, 16, sectorial planar and columnar; 17, plate-like skeleton rib-like.

These groups of forms were arranged on the basis of discrete signs, i.e., by the presence or absence of each from $22^{\text {th }}$ morphological properties of crystal [Kolomyts, 1976, 1977]: the dominant growth by axis $c$ or $a$, the major type of facets, the presence or absence of crystal polarity, open cavities, the step structure of facets, sectorial excrescences, etc.

The well-known method of numerical taxonomy was used for systematization, making it possible to represent the crystal shape in quantitative form. Numerical taxonomy was based on the dichotomous principle with association or similarity coefficients $S$ calculated by the formula [Bailay, 1967]:

$$
\mathrm{S}=\left(\mathrm{n}_{11}+\mathrm{n}_{00}\right) / \mathrm{N},
$$


where $N$ - the total number of signs, $n_{11}$ and $n_{00}$ - the number of signs present and absent in both taxons, respectively. The matrix of coefficients of similarity of the taxonomic units has been constructed on the basis of calculations (Table 5). It clearly shows 5 groups of taxons: integral wholes within which individual crystals have $S \geq 0.7$ (no less than $70 \%$ significance level of similarity). These groups have been designated as the classes of crystalline forms (see above), which are similar in the dominant faceting elements (facets, edges, tips) and in the degree of external manifestation of the inner symmetry of individual crystals. The classes of crystal forms have the following names: (1) fragmentation stage, (2) polyhedral, (3) faceted, (4) semi-skeleton, and (5) a complex of skeleton classes. The classes are grouped into three genetic types of deposited dry snow. The first and second classes are referred to the types of primary idiomorphic and isomorphic snow, respectively; other classes are referred to the type of secondary idiomorphic snow.

The complex of skeleton crystals is characterized by the greatest diversity of shapes and degrees of similarity between them $(S=0.64-0.91)$. It can be divided into five groups with significance level of characteristics exceeds $75 \%$. The first and second groups form the properly skeleton class as the highest stage of crystalline forms. Compositional and degrading skeleton forms are referred to the sectorial class (third group), while the forms that have completely lost visible skeleton contours but preserved skeleton rib-like growth type are referred to the planar class.

Based on the thermal conditions of snow pack recrystallization, it is possible to single out the second major taxonomic category: the type of crystalline forms. The guideline attribute for differentiation of the types is the direction of prevailing crystal growth (by axis $c$ or $a$ ). It is accompanied by a number of concomitant morphological characters: the presence or absence of caverns and cavities, the degree of crystal polarity, predominance of basal pinacoidal, prismatic, or pyramidal facets, their commensurability, etc. There are two main types of the forms: planar and columnar. They are "through" taxons since they are traced in nearly all classes of the forms. Compared to the classes, the types of forms have altogether lower values of $S(0.68-0.73)$.

Two described ordinates: classes and types of forms, have been used for construction of the crystal-morphological classification of snowpack (Table 6). This classification makes it possible to extract a much greater wealth of data on the internal processes occurring in the snowpack in comparison with those analytic techniques that are based on traditional concepts concerning the granularity of snow particles.

Table 5. Matrix of likeness coefficients of taxonomic units

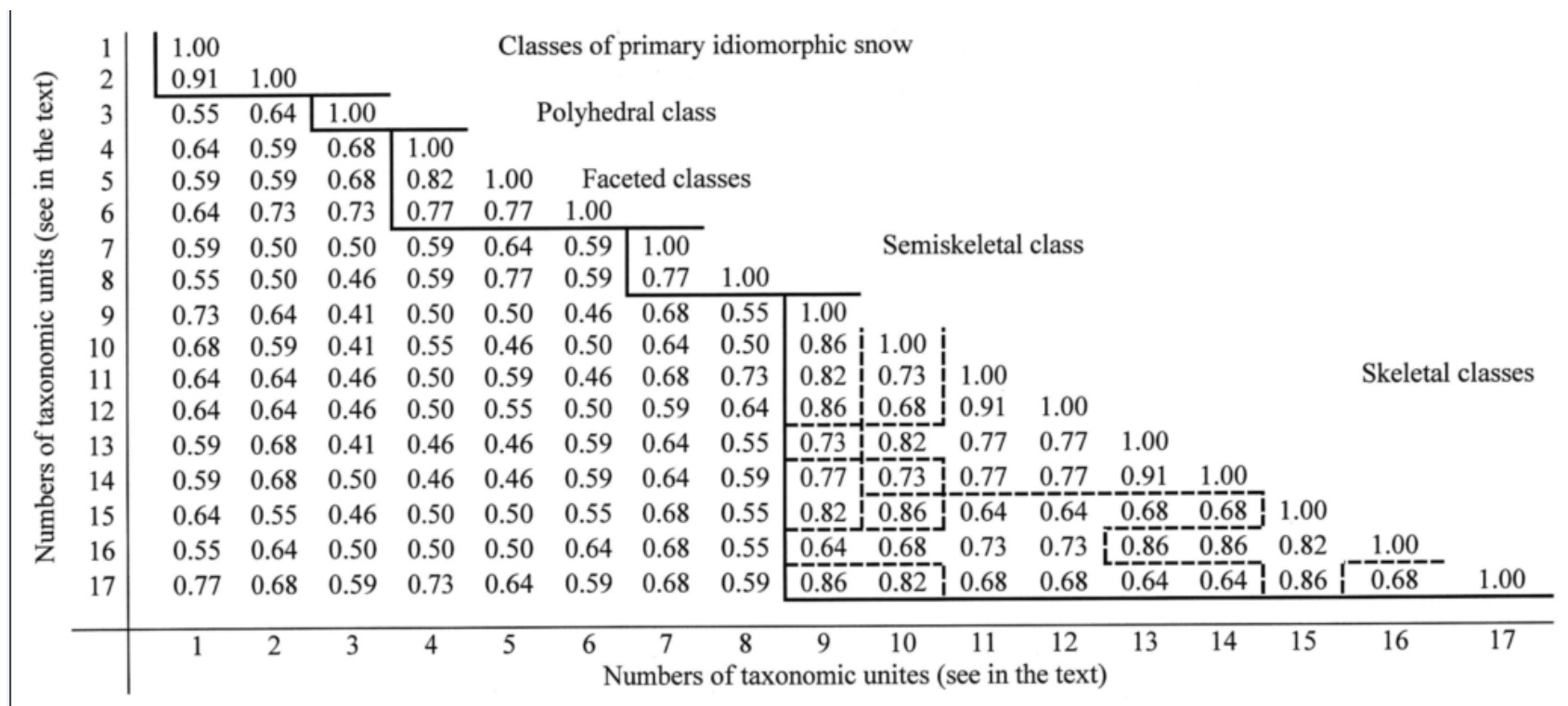

Footnote. Boundaries of crystal form classes are marked in unbroken lines; dotted lines divide the subdivisions of skeletal class complex. 
Table 6. Crystal-morphological Classification for Snow on the Ground

\begin{tabular}{|c|c|c|c|c|c|c|}
\hline $\begin{array}{l}\text { Genetic type of } \\
\text { snow }\end{array}$ & Crystal surface & \multicolumn{2}{|c|}{ Crystal form classes } & $\begin{array}{c}\text { Crystal form } \\
\text { types }\end{array}$ & Symbol & $\begin{array}{c}\text { Conventional } \\
\text { designations }\end{array}$ \\
\hline \multirow{4}{*}{$\begin{array}{c}\text { Primary } \\
\text { idiomorphic } \\
\text { snow (new-fallen } \\
\text { and old snow) }\end{array}$} & \multirow{4}{*}{ Hypidiomorphic } & \multirow{2}{*}{\multicolumn{2}{|c|}{ Faceted }} & Planar & \multirow{3}{*}{$\mathrm{I}_{0}$} & \multirow{3}{*}{$x$} \\
\hline & & & & Columnar & & \\
\hline & & Skeletal & $y$-crystal & Planar & & \\
\hline & & & & un/d * & $\mathrm{I}_{1}$ & $\rightarrow$ \\
\hline \multirow{4}{*}{$\begin{array}{l}\text { Isomorphic snow } \\
\text { (newly-deposited } \\
\text { and } \\
\text { metamorphosed) }\end{array}$} & \multirow{4}{*}{ Allotriomorphic } & Regelation- & \multirow{2}{*}{ polyhedral } & \multirow{2}{*}{ Isometric } & \multirow{2}{*}{$\mathrm{I}_{0}$} & 0 \\
\hline & & Corrasion- & & & & $\otimes$ \\
\hline & & \multirow{2}{*}{\multicolumn{2}{|c|}{ Sublimation-polyhedral }} & Planar & $\mathrm{III}_{3}$ & 0 \\
\hline & & & & Isometric & $\mathrm{I}_{2}$ & a \\
\hline \multirow{12}{*}{$\begin{array}{c}\text { Second- } \\
\text { idiomorphic } \\
\text { snow } \\
\text { (metamorphosed, } \\
\text { depth hoar) }\end{array}$} & \multirow{12}{*}{ Hypidiomorphic } & \multirow{2}{*}{\multicolumn{2}{|c|}{ Faceted }} & Planar & $\mathrm{II}_{1}$ & ש \\
\hline & & & & Columnar & $\mathrm{II}_{2}$ & 口 \\
\hline & & \multirow{3}{*}{\multicolumn{2}{|c|}{ Semi-skeletal }} & Planar & $\mathrm{II}_{3}^{\mathrm{P}}$ & ש \\
\hline & & & & Columnar & $\mathrm{II}_{3}^{\mathrm{C}}$ & $\Delta$ \\
\hline & & & & un/d & $\mathrm{II}_{3}$ & $\Delta$ \\
\hline & & \multirow{3}{*}{\multicolumn{2}{|c|}{ Skeletal mono-crystal }} & Planar & $\mathrm{II}_{4}^{\mathrm{P}}$ & $\nabla$ \\
\hline & & & & Columnar & $\mathrm{II}_{4}^{\mathrm{C}}$ & $\square$ \\
\hline & & & & un/d & $\mathrm{II}_{4}$ & $\triangle$ \\
\hline & & \multirow{3}{*}{\multicolumn{2}{|c|}{ Sectorial }} & Planar & $\mathrm{III}_{1}^{\mathrm{P}}$ & $\triangleq$ \\
\hline & & & & Columnar & $\mathrm{III}_{1}^{\mathrm{C}}$ & $\triangleq$ \\
\hline & & & & un/d & $\mathrm{III}_{1}$ & 으 \\
\hline & & \multicolumn{2}{|c|}{ Plate-like } & Planar & $\mathrm{III}_{2}$ & $=$ \\
\hline
\end{tabular}

$*$ un/d - undivided form types.

New classification has true evolutionary character, so far as it reflects successive stages of the forms of crystal growth and destruction as a result of persistent process of superposition of their crystal-chemical symmetry and dissymmetry of the hydrothermal fields and of field one-side pressure in snow-pack. It reflects also influence of external environment (the whole soil-snow-air system), which determines the type metamorphic trajectory (columnar, planar or mixed) in some or other snow layer. Thus, this classification may be used as tools instrument for the working out the long-time predictions of transition of snow pack into potentially avalanche-hazardous state. In contrast to the known physical classifications of snow, crystal-morphological classification puts an emphasis on disintegration of secondary idiomorphic snow just because its taxonomic categories make a basis of snow cover structure.

\section{Monitoring of Avalanche-Hazardous Development of Snow Layers}

In the context of avalanche hazard, the maximum attention during the study of snow sections is usually focused on the appearance and development of deep hoarfrost skeletal crystals creating the "weak layers" in snowpack. Let us trace the increase in percentage (potency) of the skeletal class of forms in each of the five snow layers considered above (see the previous section). It will be a kind of discrete monitoring of the structural state of snowpack, allowing estimation of the rate and limits of increase in the quantity (potency) of the class of skeletal crystal forms in the layers of different age and height. With that end in view, we will use the methods of calculating the rates of growth (reproduction) of populations described in the known fundamental work on ecology [Odum, 1983]. As has already been mentioned, the general trajectory of sublimation-metamorphic cycle has a logistic trajectory, where the ascending exponential part is occupied by the stages of growth of semiskeletal and skeletal crystals, while the subsequent plateau-like part of the curve is associated with regressive metamorphism. The exponential and more complete sigmoid curves of increase in the quantity of community (under non-limiting environmental conditions) are schematically presented in Figures 8, $A$ and 8 , 
$B$, respectively.

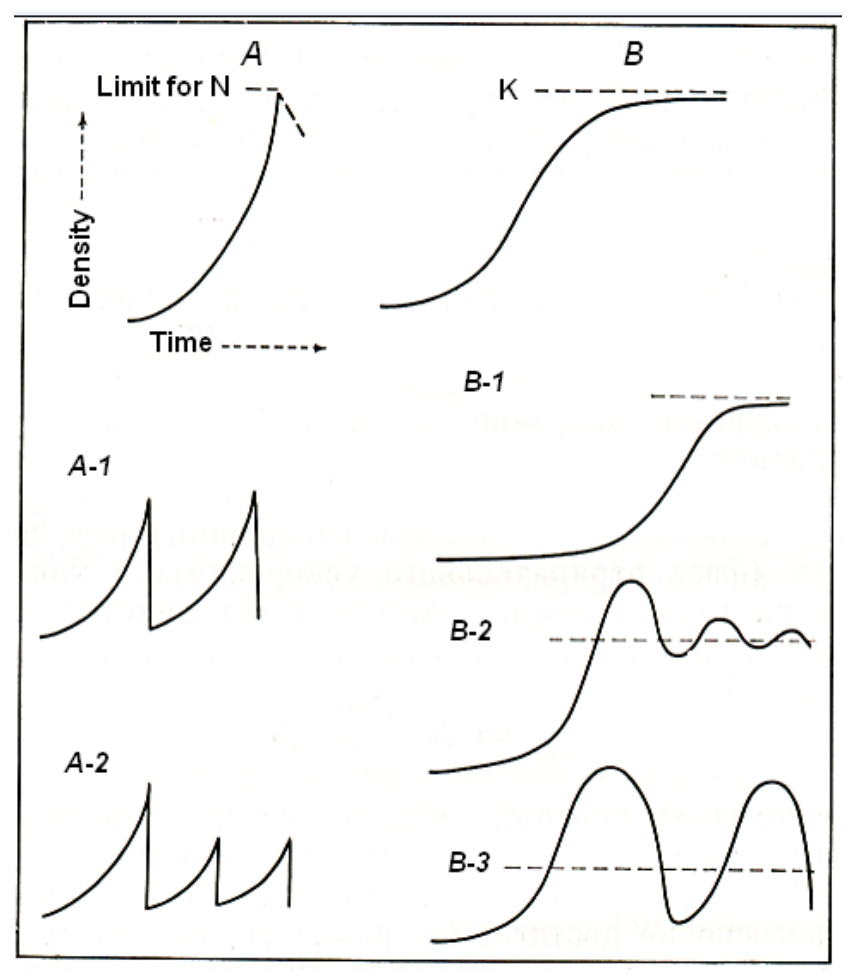

The curves of increasing of class crystal shape capacity: $A$ - exponential, for rising branch of constructive metamorphism; $B$ - S-likely (sygmoidal growth), on the whole along all trajectory of cublimation metamorphic cycle. Breachs (interruptions) in growth of class shape capacity caused either by metamorphism autoregulation (by cycles of growth buildup of layer structure) or by sharp change of hydrothermal regime in layer (the regulation from without): $A_{1}, A_{2}$ - for exponential growth; $B_{1}-B_{3} \quad$ - for sygmoidal growth.

Figure 8. Some forms of growth trajectories of crystal communities at snow layers have been represented in linear scale, by analogy with dynamics of population growth, according to [19].

Let us enumerate the main dynamic parameters. First of all, the coefficient $r$ of increase in potency of the skeletal class of forms by the moment of time $t$, i.e., "instantaneous growth coefficient" (Odum's terminology) of a given crystal community, is determined:

$$
\begin{array}{r}
N_{t}=N_{0} \cdot e^{r t} ; \\
r=\left(\ln N_{t}-\ln N_{0}\right) / t .
\end{array}
$$

Here, $N_{0}$ and $N_{t}$ are the quantity (potency) of the skeletal class in the initial and $t$ moments of time, respectively. The value of growth rate coefficient $r_{1}$ is calculated for each time interval between the two moments of structural investigation of snow profiles.

The ratio $r_{1} / r_{\max }$ between the specific and maximum growth rates of the skeletal class $\left(r_{\max }-\right.$ the highest reproductive potential), which is observed in the given snow profile, characterizes the limiting effects of hydrothermal and pressure fields in some or other layer on snow structure evolution. This ratio describes the so-called environmental resistance, according to (Odum, 1986). The following two important dynamic parameters are of interest as well: (1) $r_{2}$, the coefficient of the final rate of potency increment of the skeletal class of forms by the "preset" exponential branch of metamorphism (in essence, it is the predictive parameter of growth rate) and (2) the number of days $T$ necessary for doubling the quantity of skeletal crystals at a given rate of their growth. The above parameters are calculated by the formulas:

$$
\begin{gathered}
r_{2}=\operatorname{antilog}_{e} r_{1} ; \\
T_{1}=\log _{e} 2 / r_{1}=0,6931 / r_{1} ; \\
T_{2}=\log _{e} 2 / r_{2}=0,6931 / r_{2} .
\end{gathered}
$$

If we consider the logistic (sigmoid) trajectory of metamorphism as a whole, for each $i$ step of this trajectory it is possible to calculate the average rate $\Delta N_{i} / \Delta t$ of increase or decrease in the quantity of skeletal forms by the formula, according to [Odum, 1983]:

$$
\Delta N_{i} / \Delta t=r_{1} N_{i}\left[\left(N_{\max }-N_{i}\right) / N_{\max }\right],
$$

where $N_{\max }$ is the maximal power of the skeletal class of forms recorded in the given layer (the upper asymptote). The ratio $\left(N_{\max }-N_{i}\right) / N_{i}$ characterizes the correction for the skeletal growth of crystals determined by the limiting environmental effect, both internal (the phenomenon of superposition of symmetries of crystal and the ambient vaporous medium) and external (general changes in the hydrothermal and pressure fields in snowpack).

The results of calculations are given in Table 7, and they are very representative. The rate characteristics of "skeletization" of the layers of different height are unequal, with certain local deviations against the general decrease of this rate bottom-up along the snow profile, creating a rather variegated dynamic picture. Snowpack can be divided into two "floors" by the most common properties of "population" dynamics of the layers under consideration: the lower $(0-15$ $\mathrm{cm}$ ) and much thicker upper one (above $15 \mathrm{~cm}$ ).

In the thin near-ground snow layer $(0-2 \mathrm{~cm})$, the growth rates and shaping of crystals were initially rather high $\left(r_{1}=\right.$ $0.0457, \Delta N / \Delta t=0.266$, with doubling of the quantity of skeletal forms during $15 \rightarrow 11$ days). However, the total quantity of skeletal and semiskeletal crystals by early December was no more than $20 \%$ and the total thickness of snowpack hardly reached $30 \mathrm{~cm}$. For this period with such snow thickness it is hardly possible to suggest the appearance of a serious avalanche hazard [Bozhinsky, Losev, 1978].

Later on, there was a transition to regressive metamorphism, which substantially weakened the process of "reproduction" of forms of the skeletal class (reproductive potential $r_{1} / r_{\text {max }}$ dropped more than two times, with $\Delta N / \Delta t$ $\rightarrow 0$ ), thereby cancelling the possible transition of the near-ground layer to the "weak" state. From the third decade of January to the pre-spring period, the quantity of skeletal crystals progressively decreased $(\Delta N / \Delta t=-0.093)$, reducing approximately twofold first every 36 days and then every 16 days. 
Table 7. Myao-Chan range (Lower By-Amur). Middle-mountain silver/fir-spruce forest on the south-east macrposlope. Parameters of increasing or decreasing velosity of relatively number for crystal shapes of skeleton class (see in the text) in different snow layers (winter 1976/77)

\begin{tabular}{|c|c|c|c|c|c|c|c|c|c|}
\hline \multirow{2}{*}{$\begin{array}{l}\text { Height } \\
\text { of layer, } \\
\mathrm{cm}\end{array}$} & \multirow{2}{*}{$\begin{array}{l}\text { Period } \\
\text { of time }\end{array}$} & \multirow{2}{*}{$\begin{array}{c}\text { Number } \\
\text { of days } \\
(t)\end{array}$} & \multicolumn{5}{|c|}{$\begin{array}{l}\text { By exponential curve } \\
\text { (see Fig. } 6.11, A \text { ) }\end{array}$} & \multicolumn{2}{|c|}{$\begin{array}{c}\text { By sygmoidal curve } \\
\text { (see Fig. } 6.11, B \text { ) }\end{array}$} \\
\hline & & & $r_{1}$ & $r_{1} / r_{\max }$ & $r_{2}$ & $T_{1}$ & $T_{2}$ & $N_{\max }, \%$ & $\Delta N / \Delta t$ \\
\hline \multirow{3}{*}{$0-2$} & $24 / \mathrm{X}-4 / \mathrm{XII}$ & 42 & 0.0457 & 0.80 & 0.0659 & 15.2 & 10.5 & & 0.266 \\
\hline & $4 / \mathrm{XII}-21 / \mathrm{I}$ & 48 & 0.0211 & 0.37 & 0.0374 & 32.8 & 18.5 & 22 & 0 \\
\hline & $21 / \mathrm{I}-21 / \mathrm{III}$ & 59 & -0.0194 & -1.00 & -0.0447 & -35.7 & -15.5 & & -0.093 \\
\hline \multirow{3}{*}{$10-15$} & $29 / \mathrm{X}-4 / \mathrm{XII}$ & 37 & 0.0392 & 0.69 & 0.0567 & 17.7 & 12.2 & & 0.172 \\
\hline & $4 / \mathrm{XII}-21 / \mathrm{I}$ & 48 & 0.0438 & 0.77 & 0.0631 & 15.8 & 11.0 & 41 & 0 \\
\hline & $21 / I-21 / I I I I$ & 59 & -0.0047 & -0.24 & -0.068 & -147.5 & -100.5 & & -0.036 \\
\hline \multirow[t]{2}{*}{$30-34$} & $4 / \mathrm{XII}-21 / \mathrm{I}$ & 48 & 0.0517 & 0.91 & 0.0757 & 13.4 & 9.2 & 21 & 0.241 \\
\hline & $21 / \mathrm{I}-21 / \mathrm{III}$ & 59 & 0.0069 & 0.12 & 0.0118 & 100.4 & 61.3 & & 0 \\
\hline \multirow[t]{2}{*}{$45-47$} & $6 / \mathrm{XII}-21 / \mathrm{I}$ & 46 & 0.0568 & 1.00 & 0.0851 & 12.2 & 8.1 & 26 & 0.350 \\
\hline & $21 / \mathrm{I}-21 / \mathrm{III}$ & 59 & 0.0082 & 0.14 & 0.0152 & 84.5 & 45.6 & & 0 \\
\hline \multirow[t]{2}{*}{$60-65$} & $25 / \mathrm{XI}-21 / \mathrm{I}$ & 57 & 0.0393 & 0.69 & 0.0569 & 17.6 & 21.2 & 15 & 0.115 \\
\hline & $21 / \mathrm{I}-21 / \mathrm{III}$ & 59 & 0.0046 & 0.08 & 0.0066 & 150.7 & 105.0 & & 0 \\
\hline
\end{tabular}

Footnote. Figure with sign «-》 show that number of skeletal crystals decrease. Value $\Delta N / \Delta t=0$ testifys that logistic curve of the grouth had alredy gone out " plateau".

The longer period of constructive metamorphism took place in the overlying layer of $10-15 \mathrm{~cm}$, where almost till the end of January there was a progressive increase in the quantity of skeletal crystals $\left(r_{1}=0.0392 \rightarrow 0.0438 ; r_{1} / r_{\max }\right.$ $=0.69 \rightarrow 0.77)$, though the total growth rate of the latter was slightly lower than in the near-ground layer in the first 1.5 months of winter (the initial value $\Delta N / \Delta t=0.172$ ). More than 85-day duration of skeletal growth, with a rather high rate of doubling of the potency of this class of forms $\left(T_{1}=\right.$ 17.7-15.8 days), resulted in the fact that this layer in the third decade of January possessed the maximum (for the whole winter and for the whole snow profile) quantity of skeletal "reberniks" (about 41\%). At the same time, the latter were solely of the columnar type, which contributed to the development of fragile fibrous texture. In this winter period, the snow layer developed as the most avalanche-hazardous one. Though, in the calculations we did not take into account the stabilizing effect of age-specific build-up of the layer structure; therefore, the $r_{2}$ and $T_{2}$ values should be considered slightly overestimated and underestimated, respectively. Nevertheless, obviously it was just the snow layer at a height of $10-15 \mathrm{~cm}$ that could in the first instance pass into the critical state.

Then regressive metamorphism began to manifest itself here as well $\left(r_{1}=-0.0047 ; \Delta N / \Delta t=-0.036\right)$, but the tempos of its development were insignificant $\left(T_{1} \rightarrow T_{2}=(-148) \rightarrow\right.$ $(-100)$ days), and it caused only a minor reduction in the quantity of skeletal crystals (to $31 \%$ ). It seems that this layer remained avalanche-hazardous till the end of winter, the more so that the total thickness of snowpack by March 20 reached $110 \mathrm{~cm}$.

As we can see, the most rapid transition into the "weak" state may not be observed in the lowest, near-ground snow layer. Progressive development of such state in the snow layer requires quite definite hydrothermal conditions (temperature, temperature gradient, and the density of macroscopic vapor diffusion), and this problem needs further investigation. As has already been mentioned, the cases of avalanching not along the near-ground snow layer but along the layers lying at a certain height above the ground have been more than once observed in avalanche research practice.

The overlying "floor" of snowpack, being almost wholly a little younger than the 10-15-cm layer, did not go beyond the limits of constructive metamorphism till the end of winter; therefore, the sufficiently long skeletal growth (about 60 days) imparted a certain potential of avalanche hazard to the layers at heights of 30-34 $\mathrm{cm}$ and $45-47 \mathrm{~cm}$. However, the active growth of potency of the skeletal class $\left(r_{1}=\right.$ $0.0517-0.0568 ; \Delta N / \Delta t=0.241-0.350)$ was typical here only of the first half of winter, when the quantity of its forms was no more than 11-16\%. In addition, constructive metamorphism occurred by the mixed variant, when massive development of planar skeletal crystals could prevent the appearance of avalanche-hazardous columnar-fibrous texture (see above). At the same time, the layer at a height of $45-47 \mathrm{~cm}$ left behind all underlying layers as regards the tempos of "skeletization" in this period (6/XII-21/I), namely due to considerable prevalence of planar skeletal growth over columnar growth.

In February-March, skeletal growth over the whole upper "floor" of snowpack abruptly slowed down (with the signs of sectorial growth); hence, the $r_{1}$ value proved to be lower by an order of magnitude than before and the sigmoid growth curve reached a plateau $(\Delta N / \Delta t \rightarrow 0)$. The planar variant of metamorphism developing almost solely in the upper snow 
layer at $60-63 \mathrm{~cm}$ caused a rather quick appearance of the first generation of skeletal crystals $\left(r_{1}=0.0393\right)$; however, the final share of the skeletal class of forms did not exceed $15 \%$ because of further slowdown of the process.

We have considered the "population" dynamics of snowpack layers of different age and height, using their simplest structural character: the quantity of the skeletal class of forms, which is usually taken to determine the "weak" layers. If the fibrous texture of such layers is composed not by skeletal but by faceted (as a rule, columnar) crystals, then the avalanche hazard should be monitored by the dynamics of capacity of this class of forms. The presented dynamic characteristics of the structure, with involvement of additional mechanical parameters of snow (in particular, compression strength and resistance to subhorizontal shift) can be probably used for predicting the time of transition of some or other layer into avalanche-hazardous state. Analogous dynamic characteristics can be also calculated by the complex structural parameter of snow layer $-P_{\bar{O}}$, which will give more comprehensive information for prediction of the places and times of appearance of critical states in snow pack.

\section{Mechanisms f Auto-Regulation of Metamorphism in Snow Layers}

The deterministic model of the sublimation-metamorphic cycle is a result of its ultimate schematization (abstraction); therefore, it cannot reflect the whole diversity of metamorphic processes. Such diversity is conditioned by probabilistic character of metamorphic processes exhibited in the mechanisms of their auto-regulation [Kolomyts, 1984]. Any evolution is at last the auto-regulation [Ashby, 1956;
Shmalgause, 1968]. These processes include two basic types of regulation of snow layer metamorphism (Figs. 9 and 10): (1) self-regulation - "motion" of the layer according to one of the "set" programs for the trajectory of the metamorphic cycle and subsequent growth "buildup" of the structure; (2) their regulation from outside - under the influence of atmospheric perturbations (warming and cooling periods, snowfalls, snowdrift phenomena, etc.) which transfer the layer from one program of development to another and thereby accelerate or, conversely, retard the overall rate of metamorphism. The key task in analyzing the evolution of a snow pack is clearly demarcating these two basic processes because only then is it possible to arrive at a practicable forecast of the critical state of the structure of some layer [Kolomyts, 1984].

The mechanisms of auto-regulation of snow layers may be traced in the transitions of each one of them from one state to another, that is, when comparing structural changes which have occurred during a given step of time. Such transitions in principle are probabilistic in character, this being due to the non-uniform growth of different crystals of the same age. The "motion" of the first growth generation of depth hoar crystals on the trajectory of the sublimation-metamorphic cycle is accompanied by a successive increase in the number of classes of crystal forms to some maximum values. In this, however, the transformation of the layer does not stop even under conditions of a steady-state external medium. With the appearance of a substantial quantity of skeletal forms, there begin cycles of growth buildup of sets of single crystals. In addition, when in a state in which skeletal forms of "mature" depth hoar dominate, the layer during two or three successive steps may transition to a new program of re-crystallization if an abrupt, stable change in the conditions of the external medium has occurred.

Table 8. Example of matrixes of real transformation in form of depth hoar crystals in any layer of snowpack $(A)$ and of transformation crystal forms in the same layer during Markov's process $(B)$. Step length is $25-35$ days
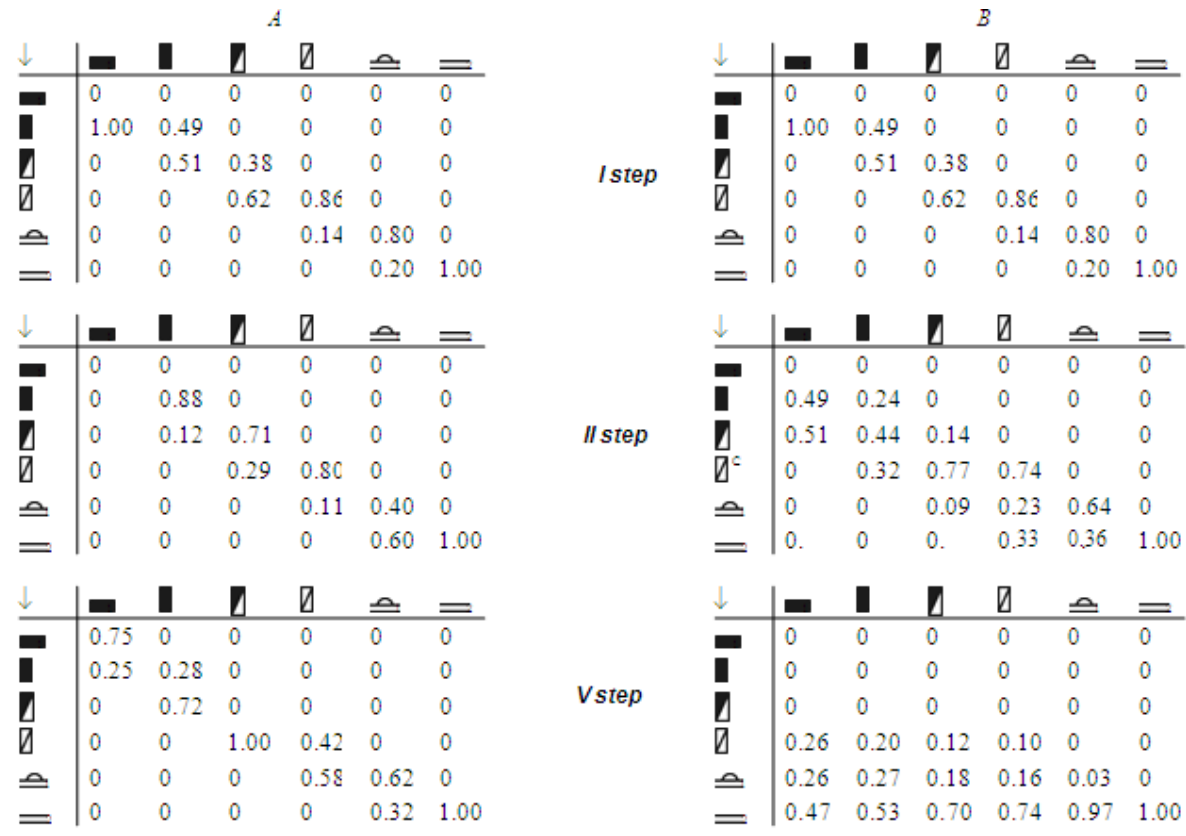


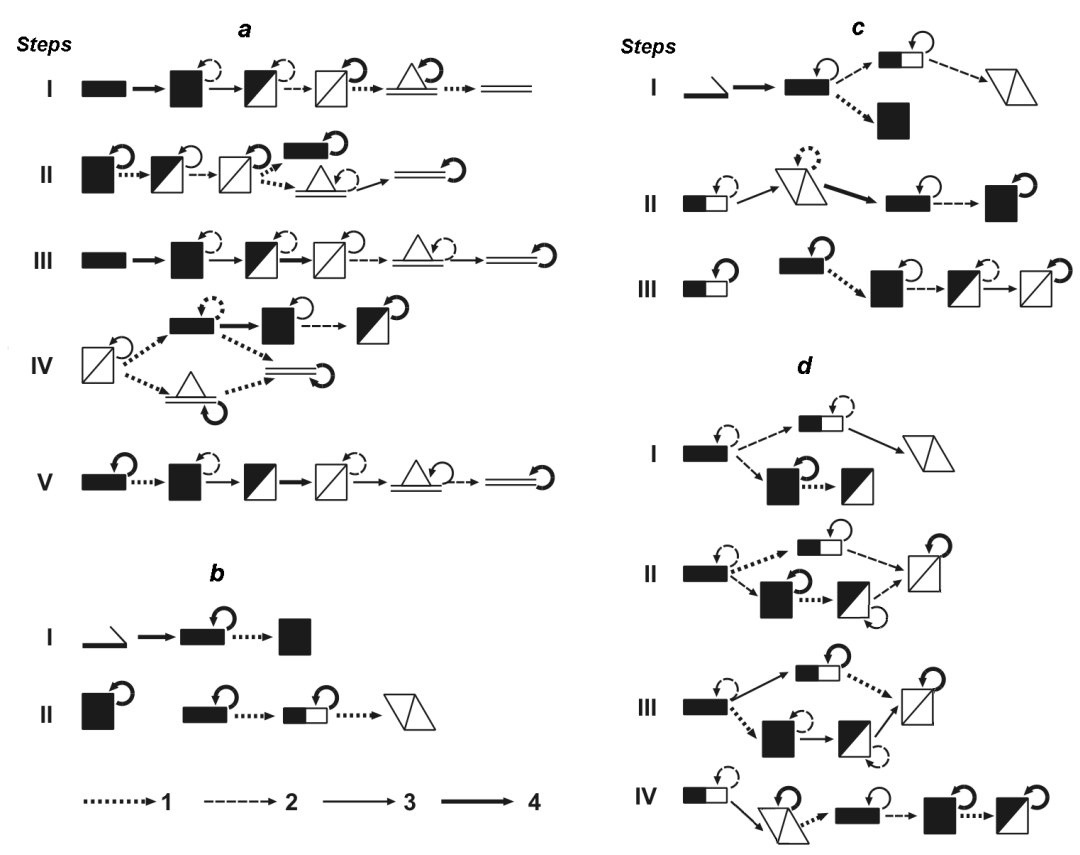

Models of self-regulation (self-development): $a$ - a complete columnar program; traced are the linear cycle of transformations of crystal shapes and two cycles of growth complexity in the structure of the layer; $b$ - incomplete planar program; columnar faceted forms of skeletal single crystals are not produced. Models of the regulation of metamorphism from outside: $c$ - polygenetic program with one irreversible perturbation from the planar algorithm to the columnar; $d$ - polygenetic program with simultaneous planar and columnar crystal growth; planar skeletal growth begins earlier and advances more rapidly than the columnar. Probabilities (frequency) of transitions of one form classes into another: $1-0.01-0.25 ; 2-0.26-0.50 ; 3-0.51-0.75 ; 4-0.76-1.00$. 1 step $\approx 25-35$ of days. The remaining symbols are defined on Fig. 4, $B$, and in Table 6.

Figure 9. Stochastic structural models of the self-regulation in the sublimation metamorphism of snow layers.

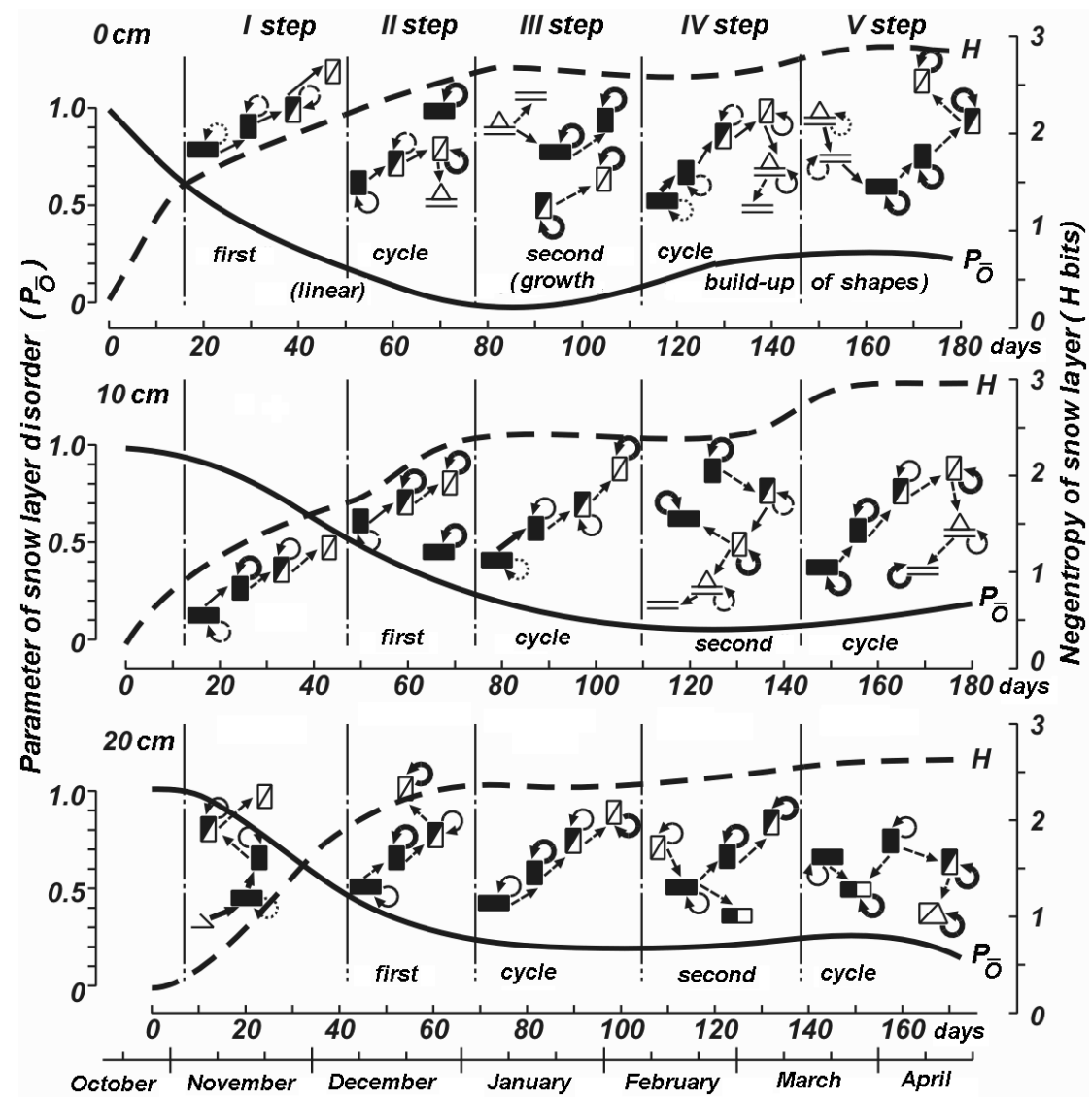

Figure 10. Seasonal dynamic of complexity and disorder measures of layers in snow pack of primary dark/coniferous-taiga natural complexes (middle taiga of West Siberian, winter 1968/69). 
The processes of auto-regulation of metamorphism of snow layers are disclosed with the aid of matrices and graphs of the probabilities of transitions of crystal classes and types of forms into others during a given series of time steps (see Table 8, Figs. 9 and 10). Methods of calculation of such transitions are described in [Ashby, 1956]. Modeling was based on the materials of regime surveys carried out by the authors at experimental test site in middle taiga of West Siberian [Kolomyts, 1976].

\subsection{The Process of Self-Regulation in the Metamorphic Cycle}

In the lower snow pack layers, which usually have the highest rate of re-crystallization, the assemblage of crystals over the period of the first two steps traverses practically the entire elemental chain of transformations in accordance with the columnar algorithm (see Fig. 9, a). "The center of gravity" of the transitions is concentrated in the skeletal forms. Further transformations of snow layer are associated with the growth build-up of the capacity of classes of crystal forms, the process of which is fluctuating and cyclical in character externally resemble the "population waves" known from the evolutionary biology [Odum, 1983].

Each cycle begins with the break in the elementary chain after the stages of faceted prisms or between them. All of the most developed faceted crystals are exhausted during intensive skeletal growth. We can say that the first suppliers of raw material for evolution are consumed, using the figure of speech from the theory of evolution [Odum, 1983]. Other individuals of faceted classes are still within their growth stages and cannot supplement the "emergency stock" of the crystal community. There follows a deficit of faceted crystals prepared (in accordance with their kinetic resistance) to skeletal growth, which causes the chain rupture. Direct skeletal growth of new crystals is not bulk and therefore cannot cover the deficit. The latter is neutralized mainly due to the appearance and development of a new (second) generation of crystal communities, beginning from plane faceted prisms, partially substituting for the skeletal and lamellar shapes (the second and forth steps in Fig. 9, a). That entails a new noticeable increase in the measure of complexity $(H)$ and decrease in the parameter $P_{\bar{O}}$ of the layer (see Fig. 10).

The "wave" of the crystal shortage in each cycle traverses over the entire elemental chain to cause a series of corresponding reactions in each of its links. The cycle ends with the complete restoration of the elemental chain and renewal of intensive skeletal growth (third and fifth steps in Fig. 9, a). In each cycle of crystal buildup the snow layer returns as it were to the initial stages of constructive metamorphism. The periodic "rejuvenation" of the crystal assemblage of the snow layer, due exclusively to its self-development, may substantially change the mechanical properties of the snow even with unchanged external conditions. Since the feedback is slightly delayed relative to the direct perturbation, the cycles of age-related layer complication appear as pulsating pendulous oscillations, periodically moving away from the final state and then approaching it. After each cycle, the phase state of the layer is shifted to the right along the elementary chain of the sublimation-metamorphic cycle (see Fig. 10), i.e., approaches the state of structural stability. There is an obvious picture "... of interruptive-continuous development of mineralogical processes" [Yushkin, 1977, p. 111].

The "waves" of self-regulation are the phenomenon of the stabilizing selection of crystal forms, which contributes to elongation of the period of constructive metamorphism in snow layer and hinder from their transition to regressive metamorphism by cycles of periodical age-specific build-up of its structure. The effect of the stabilization is traced while comparing real steps in the evolution of the snow layers (see Table $8, A$ ) with the steps of a computational "Markov's machine" [Harbaugh and Bonham-Carter, 1970], where all relative frequencies of the transitions are specified according to the first step (see Table 8, B). The steps of a Markov regulating operation are obtained by sequentially multiplying the matrix by itself. This computation makes it possible to forecast the final state of the system with the initial frequencies of transitions preserved in all steps.

In a Markov regulating operation the snowpack should attain states towards the end of winter when the probabilities of final crystal forms and of transitions to these forms become almost identical and close to 1 . In facts, thanks to the stabilization effect, the layers usually do not attain these states. The difference in frequencies of transitions between the real and the Markov's processes of self-regulation of metamorphism is that corrective measure which should be introduced when forecasting the structure of the snow layer at each subsequent time step.

Because the growth of skeletal crystals directly from planar faceted prisms is characteristic of the planar variant of metamorphism, a significant quantity of skeletal forms may appear in a month (see Fig. 9, b). Columnar faceted forms grow extremely slowly, gradually lose the base of their development and degrade. The planar variant of growth differs from the columnar by the large nonuniformity of crystal growth in the development of crystals of the same growth generation, this conforming to the more complex hydrothermo-dynamic fields of snow. Dominant in this case are the prolonged stabilization of forms and the weak intensity of the transitions, thanks to which the elemental chain of the plot is preserved over several steps.

With each new cycle of "rejuvenation" of crystal shapes, the layer is shifted to the initial phase of constructive metamorphism and once again passes through all of its subsequent phases. Consequently, age-related complication of the layer may be considered as a form of its self-regulation aimed at stabilization of the structure in the framework of progressive development and at complete or partial prevention of the phases of regressive metamorphism resulting in elimination of hypidiomorphic particles. This process is determined by immanent "aspiration" of growing crystals for the integrity of their shapes in the course of 
superposition of their internal high order symmetry and dissymmetry of the growth-supporting microenvironment.

As we can see, there is a rather distinct analogy between the "life" of crystal communities in snow layer and organic life [Shmalgausen, 1969], which allows us to consider such communities as a kind of ecological systems possessing a certain compensating - stabilizing mechanism, according to [Odum, 1983].

\subsection{Regulation of snow Metamorphism From Outside}

The processes of self-regulation in the snowpack layers periodically become» complicated by external perturbations, with subsequent restructuring of the current program of structure transformations from planar to columnar or the reverse. External regulation is responsible for adaptive selection of crystal forms in snow layers. The most frequent type of perturbations is characteristic of layers which have remained a long time near the snow surface during severe cooling and sudden fluctuations in temperature and then were buried under new thick layers of snow and located in more moderate temperature conditions. A similar effect may be produced also by prolonged stable warming. Such external perturbation is registered in the second step of transitions (see Fig. 9, c). A sudden perturbation in the algorithm causes the breakup and subsequent extinction of the planar chain of development. The number of beveled skeletal forms is reduced catastrophically, and the "center of gravity" of the transitions displaces to faceted columnar prisms which already reveal signs of skeletal growth. However, snow layer "thrown back" two entire steps by perturbation (from skeletal to faceted columnar) may never attain the state of "mature" depth hoar.

Often observed in the upper layers of the snow pack are mixed variants of metamorphism with simultaneous development of both planar and columnar types of crystal forms. When the temperature field in the snow layer is manifestly non-steady state, the planar chain of transitions significantly outstrips the columnar (see Fig. 9, d). Subsequently when the columnar types reach the skeletal phase of growth both chains converge. Pulsating "waves" of shortages of forms alternately pass through each chain, causing corresponding attenuation and amplification of the transition frequencies. With an overall increase in temperature at the end of winter the planar chain usually decomposes and the "center of gravity" of the transitions shifts to columnar types of forms with a slowing of the processes of metamorphism.

On the whole if the transition of a snow layer from the columnar program of crystal growth to the planar somehow accelerates the process of "maturing" of the depth hoar, then a reverse shift from the planar algorithm to the columnar leads to slowing of this process. On its basis one should correct for the effect of external perturbations when predicting the structure of different layers of the snow pack. The multiple reprogramming of crystal growth substantially slows down the general process of sublimation metamorphism. Formation of a new chain needs time; therefore, subsequent transition frequencies decrease compared to the initial ones. In addition, the growth of the first generation of crystals comes to a halt during perturbations. The cycles of age-related complication of the layer begin even before it reaches the skeletal phase, which also hampers the layer evolution. It is obviously the need for introducing certain corrections to the trajectory of self-development of crystal communities with allowance for the effect of external (atmospheric) perturbations when predicting the structure of some of other snowpack layers for more precise determination of the real time of their transition to the avalanche-hazardous state.

\section{Conclusion}

1. The crystal-morphological and symmetry studies of snow packs made it possible to go deep into the essence of mechanisms of sublimation metamorphism, to estimate the role of internal and external factors of snow re-crystallization and, finally, to arrange the conceptions of milestones of this re-crystallization. It is worked out the deterministic and probability models describing the sublimation-metamorphic evolution cycle of seasonal snow cover, the polymorphic variants of this cycle and processes of internal and external auto-regulation of metamorphism.

With respect to form the development of the structure of the snow pack obeys the same basic laws that are peculiar to biosystems. This enables us to use the known achievements of modern biology for elucidating the many processes occurring within the snow cover, relying on a fundamental tenet of cybernetics - the law of structural similarity of objects with different substrata. Some of these achievements were used in the present work, producing some particularly constructive results. On the one hand it is obviously that crystal-morphological structure of snow cover may be considered as a "quickly running" model of many evolutionary biosphere processes.

2. Evolution of each snow layer is a directed process of appearance and disappearance of successively alternating ways of spatial ordering of crystal forms under energetic influence of snow environment as a necessary condition of this evolution. The origins of the mechanisms of directed evolution of snow layers are concentrated in the crystal-medium (vapor) and crystal-crystal systems.

The main driving force of processes yielding an evolutionary row of crystals is not random variations of the environment (the near-ground air layer or soil) but internal interactions within a snow pack, which are characterized by certain autonomy. For all that the factor of time (the age of genetic horizon) plays a crucial role in sublimation metamorphism. The mechanisms of directional evolution of snow layers originate in the crystal-medium (vapor) system. Stages of the forms of crystal growth and destruction are a result of consistent process of superposition of their crystal-chemical symmetry and asymmetry of the 
environment - in accordance with the P. Curie's principle. Self-development crystal communities in snow layers is main process of structure transformation of snow.

3. Diversity of snow structures is caused both invariant directed process of stage development of crystal forms and probabilistic dynamic of crystal communities exhibited in the mechanisms of their auto-regulation. Accordingly snow cover can be considered as the evolutionary-adaptive glaciosystem. Deterministic and stochastic modeling of metamorphic processes opens a way to long-time prediction of snowpack potentially avalanche-hazardous state. Internal and external waves of metamorphism auto-regulation are inevitably associated with periodical transitions of snow layers into the state of "mature" depth hoar (mass development of skeletal forms).

The processes of self-regulation in snow layers strongly resemble the population waves known in evolutionary biology, or "the waves of life", which are suppliers of evolutionary material for relatively intensive selection. Such fluctuations influence the direction and intensity of crystal form selection: reduce it on the ascending branch of the quantity of individuals of the given class of forms and enhance it on the descending branch. Thus, these fluctuations are a factor that prolongs the period of progressive metamorphism branch in the snow layer by way of periodic age-related buildup of its structure. There is a phenomenon of stabilizing selection in the evolving community of crystal forms.

4. The proposed crystal-morphological and symmetry methods of snowpack structure analysis make it possible to extract a much greater wealth of data on the internal processes occurring in the snowpack in comparison with those analytic techniques that are based on traditional concepts concerning the granularity of snow particles.

In fact excluded from consideration in the granule-metric approach to analysis of snow structure is such a vitally important characteristic as the form of growing or vaporizing crystals (and consequently, the quantitative measure of the quality of the structure), without which it is simply meaningless to discuss the evolution of snow and the directionality of its metamorphism. The crystal-morphological and symmetry parameters examined above and not the dimensions of the grains and their contacts should serve as the main guiding signs of the different levels of metamorphism (evolution) of snow and as the basis for predicting its potential structural changes. This postulation follows from the fundamental principles of contemporary crystallography and the theory of metamorphism.

5. The essence of metamorphic transformations of snow is not in the diffusion and mechanical redistribution of solid material in a given volume of snow but in the staged changeover in the various means of spatial order in crystallized material, this being manifested first of all in two morphological signs: (1) in the staged changeover in shapes of growth or dissolution (vaporization) of the crystals; (2) in the change in their external (geometric) symmetry through successive deviation from their ideal (crystal-chemical) symmetry. The granulometric characteristics are only accessory and, moreover, are ambiguous parameters describing the dynamics of the processes of re-crystallization, and hence using them is a necessary but altogether insufficient condition for the quantitative evaluation of the structure of snow.

6. The crystal-morphological and symmetry features of snowpack structure are not only purely structural in character. Numerous investigations have proved that morphology of crystals discloses definite linkages with the mechanical properties of snow and with meteorological conditions - the very characteristics that are taken into account as avalanche-formation factors in engineering computations.

7. New morphological dynamic classification of snow crystals in snow cover has been worked out by author on the base of evolutionary conception of snow sublimation metamorphism. For the first time the method of numerical taxonomy was used for systematization, making it possible to represent the basic property of a crystal (shape) in quantitative form. Two categories of crystal forms have been picked out by matrixes of coefficients of similarity of the taxonomic units: classes and types of forms. The first category reflects the invariant aspect of snow sublimation metamorphism and the second one - the hydrothermal conditions of this process in each layer of the snowpack, that is the polymorph variants of united sublimation-metamorphic cycle. Type of crystal form is closely connected with the degree of winter continentality and the position (on the depth) of given layer in the snowpack as well.

\section{REFERENCES}

[1] Ashby, W.R. (1956). Introduction to Cybernetic. Macmillan \& Co., London.

[2] Armand, D.L., (1975). Nauka o landshaphte. Nauka, Moskva (in Russian).

[3] Bailey, N.T.J., (1967). The mathematical approach to biology and medicine. John Wiley and sons, London - New York - Sydney.

[4] Bartelt, P., Buser, O., Sokratov, S.A., (2002). A nonequilibrium treatment of heat and mass transfer in alpine snow covers. Cold Region Science and Technology, vol. 35, № 3, pp. 219-242.

[5] Bartelt, P., Lehning, M., (2002). A physical SNOWPACK model for Swiss avalanche warning. Part I; numerical model. Cold Region Science and Technology, vol. 35, pp. 123-145.

[6] Bozhinsky, A.N., Losev, K.S., (1978). Osnovy lavinovedeniya. Gidrometeoizdat Leningrad (in Russian).

[7] Bozhinsky, A.N., Nazarov, A.N., Chernous, P.A., (2002). A probabilistic model of snow ava-lanche:origin and motion. Data of Glaciol., vol. 93, pp. 79-84.

[8] Brillouin, L. (1956). Science and information theory. 
Academic Press INC • Publishers, New York.

[9] Brown, R.L., and Edens MQ (1991). On the relationship between neck length and bond radius during compression of snow. J. Glaciol., vol. 37, № 126pp. 203-208.

[10] Brun, E., David, P., Sudul, M., Brunot, G.A., (1992). A numerical model to simulate snow-cover stratigraphy for operational avalanche forecasting. J. Glaciol., vol. 38, № 128, pp. 13-22.

[11] Chernov, R.A., (2003). Vliyanie temperaturnogo rezhima snezhnoy tolshchi na obrazovanie gorizontov razryhleniya. Materialy glyatciolog. issledovany, vol. 94, pp. 100-102 (in Russian).

[12] Colbeck, S.C. (1982). An overview of seasonal snow metamorphism. Reviews of Geophys. and Space Phys., vol. 20, № 1, pp. 45-61.

[13] Colbeck, S., Akitaya, E., Armstrong, R., Gubler, H., Lafeuille, J., et al., (1990). The International Classification for Seasonal Snow on the Ground. Intern. Commission of Snow and Ice of Internat. Assoc. of Scientif. Hydrology. Working group on Snow Classification.Curie, P., (1908) Sur la symmetrie dans les phenomenas physiques. Curie P. Oeuvres. Paris, pp.118-141.

[14] Durand, Y., Brun, E., Merindol, L., Guyomarc'h, G., Lesafere, B., and Martin, E., et al. (1993). A meteorogical estimation of relevant parameters for snow model. Annals of Glaciology, vol.18, pp. 65-71.

[15] Edens, M.Q., and Brown, R.L. (1991). Changes in microstructure of snow under large deformation. J. Glaciol., viol. 37, № 126, pp. 193-202.

[16] Fierz, Ch., Armstrong, R.L., Durand, Y., Etchevers, P., Greene, E., et al., (2009). The International Classification for Seasonal Snow on the Ground. IACS, vi +67 p.p. (UNESCO, IHP-VII, Technical Documents in Hydrology, No 83), Paris.

[17] Golybev, V.N., Frolov, A.D. (1998). Modelling the change in structure and mechanical properties in dry-snow densification to ice. Annals of Glaciology, vol. 26, pp. 45-50.

[18] Gray, D.V., Male, D.H. (Eds.) (1981). Handbook of snow. Principle, processes, management \& use. Division of Hydrology, University of Saskatchewan Saskatoon, Toronto, Canada.

[19] Harbaugh, J.W., and Bonham-Carter, G., (1970). Computer simulation in geology. Wiley-Interscience, New York London - Sydney - Toronto.

[20] Grigoryev, D.P., (1965). Ontogeny of Minerals. Izrael Program for Scientific Translations, Jerusalem.

[21] Grigoryev, D.P., Zhabin, A.G., (1975). Ontogeniya mineralov. Individy. Nauka, Moskva (in Russian).

[22] Honigman, B., (1958). Gleichgewichte- und Wachstumsformen von Kristallen. Darmstadt.

[23] Kaempfer, Th.U., Sokratov, S.A., Schneebeli, M., (2004). The effect of the structural evolution of snow on the heat transfer. Proc. of the 3 th Intern. Symposium on two-phase flow modeling and experimentation. Edizioni ETS, Piza, 2, pp. $715-730$.
[24] Kobayashi, T., (1957). Experimental researches on the snow crystal habit and growth by means of diffusion cloud chamber. J. Met. Soc. Japan., vol. 75, pp. 38-47.

[25] Kolomyts, E.G., (1976). Structura snega i landshaftnaya indikatciya. Nauka, Moskva (in Rus-sian).

[26] Kolomyts, E.G., (1977). Techniques for the crystallomorphological analyais of snow structure.

[27] Nauka, Moskva. Translate from the Russian by C.E. Bartelt. (2011). Funds of the Swiss FederalInst. for Forest, Snow and Landscape Research Birmensdorf.

[28] Kolomyts, E.G., (1984). A Crystal-morphological Atlas of Snow (A Handbook for Snow-Avalanche Station). Nauka, Moskva. Translate from the Russian by C.E. Bartelt (1997). Funds of the Swiss Federal Inst. for Forest, Snow and Landscape Research, Birmensdorf.

[29] Kustler, G. (1957) ABC of information theory. Hubert P. Yockey (Ed) Information theory in Biology. Pergamon Press, London - New York - Los Angeles, pp. 5-48.

[30] Kuznetsov, V.D., (1953) Cristally i cristallizatsiya. Gostehizdat, Moskva (in Russian).

[31] Lehning, M., Bartelt, P.B., Brown, R.L., Fierz, Ch., (2002). A physical SNOWPACK model for the Swiss avalanche warning: Part III: meteorological forcing, thin layer formation and evolution. Cold Region Science and Technology, vol. 35, pp. 169-184.

[32] Nakaya, U., (1954). Snow crystals: natural and artificial. Harvard Univ. Press MA, Cambridge.

[33] Odum, E. P., (1983). Basic Ecology. Vol. 2. PhNYCh.: Saunders College Publishing, Philadephia - New York Chicago.

[34] Paffengolts, K.N., (Ed.) (1973) Geologichesky slovary. Chasty I. Nedra, Moskva (in Russian).

[35] Paulke, W., (1938). Practische Schnee- und Lawinenkunde. Verlag Julius Springer Berlin.

[36] Pielmeier, Chr., and Schneebeli, M., (2003). Developments the stratigraphy of snow. Surv. Geophys., vol. 24, pp. 389-416.

[37] Pianka, E.R., (1978). Evolutionary ecology. Second Edition. Harper and Row, Publishers, New York, Hagerstown, San Francisco, London.

[38] Quervain, M.R. de, (1958) On the metamorphism snow under constant pressure and temperature gra-dient. Intern. Assoc. Sci. Hydrol. Gen Assembly Toronto, 4, Snow and Ice. Gentbrugge, pp.225-239.

[39] Rikhter, G.D., (1955). Ispolyzovanie snega v narodnom khozyaystve i zadachi ego izucheniya. Voprosy izucheniya snega $\mathrm{i}$ ispolyzovaniya ego $\mathrm{v}$ narodnom khozyaystve. Izdatelstvo AN SSSR, Moskva, pp. 5-23 (in Russian).

[40] Schaefer, V.J., Klein, G.J., and de Quervain, M.R., (1954). The International Classification for Snow (with special reference to snow on the ground). 31. The Commission of Snow and Ice of the Internat. Assoc. of Hydrology. Associate Committee on soil and snow mechanics. Na-tional Research Council., Canada, Ottawa.

[41] Seligman, G. (1936). Snow Structure and Ski Fields. 
Macmillan and Co. Ltd. London, L.

[42] Shafranovsky, I.I., (1968). Lektcii po kristallomorfologii. Vysshaya shkola, Moskva (in Russian).

[43] Shafranovsky, I.I., Plotnikov, L.M.,1(975). Simmetriya v geologii. Nedra, Leningrad (in Russian).

[44] Sheftal, N.N., (1973). Gleichtgewichtsform und Form des idealen Einkristall. Kruistall und Technik, vol. 8, № 1-3, pp. 149-168.

[45] Sheftal, N.N., Kolomyts, E.G., (1973). Evolutsiya konechnyh form rosta kristallov $\mathrm{v}$ zavisimosti ot vkhozhdeniya sredy $\mathrm{v}$ ih sostav. Acta phys. Acad. Sci., Hangar. 33 (3-4): 335-351 (in Russian).

[46] Shmalygauzen, I.I., (1968). Kiberneticheskie voprosy biologii. Nauka, Novosibirsk (in Russian).

[47] Shmalygauzen, I.I., (1969). Problemy Darvinizma. Nauka, Leningrad (in Russian).

[48] Shneebeli, M., Sokratov, S.A., (2004). Tomography of temperature gradient metamorphism of snow and associated changes in heat conductivity. Hydrological Processes, vol. 18, № 18, pp.3655-3665.

[49] Shubnikov, A.V., (1975). Izbrannye trudy po kristallografii. Nauka, Moskva (in Russian).

[50] Shubnikov, A.V., Koptsik, V.A., (1972). Simmetriya v nauke i iskusstve. Nauka, Moskva (in Russian).

[51] Shumsky, P.A., (1955) Osnovy structurnogo ledovedeniya. Izdatelystvo AN USSSR. Moskva (in Russian).
[52] Socratov, S.A., (2001). Parameters influencing the recrystallization rate of snow. Cold Region Science and Technology, vol. 33, № 2-3, pp. 263-274.

[53] Sokratov, S.A., Troshkina, E.S., (2009). Rasvitie strukturno-stratigraphicheskih issledovany snezhnogo pokrova. Materialy glyatciolog. issledovaniy, vol.107, pp.103-109 (in Russian).

[54] Sommerfeld, R.A., and LaChapelle, E.R., (1970). The classification of snow metamorphism. J. Glaciol., vol. 9, № 55, pp. 3-17.

[55] Timofeev-Resovsky, N.V., Bovorontsov, N.N., Yablokov, A.V., (1969). Kratky ocherk teorii evolyutsii. Nauka, Moskva (in Russian).

[56] Tushinsky, G.K., Gusykova, E.F., Gubareva, V.D., (1953) Perecristallizatsiya snega i vozniknovenie lavin. Izdatelstvo MGU, Moskva (in Russian).

[57] Vernadsky, V.I., (1965) Himichesky sostav biosphery Semli i ee okruzheniya. Nauka, Moskva (in Russian).

[58] Watanabe, Z. (1980). Tensile strain and fracture of snow. J. Glaciol., vol. 26, № 94, pp. 255-262.

[59] Yablokov, A.V., Yusufov, A.G., (1976) Evolutsionnoe uchenie. Vysshaya shkola, Moskva (in Russian).

[60] Yushkin, N.P. (1977). Teoriya i metody mineralogii. Nauka, Leningrad (in Russian).

[61] Yosida Z (1966) Surface structure of ice crystal and its equlibrium from. Inst. Low Temperat.Sci. Phys. Sci. Sapporo, vol. 24, pp. 1-19. 